\title{
Exclusión social como factor de riesgo de violencia de género en la pareja
}

\section{Paola Damonti}

Universidad Pública de Navarra. Departamento de Trabajo Social

paola.damonti@unavarra.es

\section{Resumen}

Partiendo de una concepción estructural tanto de la violencia de género en la pareja como de la exclusión social, analizamos aquí el riesgo de experimentar esta violencia según diferentes intensidades y tipos de exclusión. Para ello utilizamos datos de la Encuesta de la Fundación Foessa sobre Integración Social y Necesidades Sociales 2013, cuyo objetivo es analizar la exclusión social en el Estado español, pero que, en su última edición, incluye también una batería de preguntas destinadas a detectar procesos de violencia de género (ejercida por la pareja o la expareja) de forma estandarizada. Los datos presentados se basan en 5.473 encuestas presenciales. Realizamos análisis bivariantes y multivariantes y distinguimos además según el tipo de violencia (física, sexual y psicológica). El riesgo de experimentar violencia de género teniendo como referencia la plena integración aumenta un $45 \%$ en exclusión moderada y un $82 \%$ en exclusión severa. Diferenciando por tipo de exclusión, se observan incrementos significativos en casos de conflicto social (un 460\%), exclusión de la educación (un 88\%) y de la salud (un 57\%), mientras que la exclusión del empleo, del consumo, de la política, de la vivienda y el aislamiento social no son significativas. Además, en referencia a las zonas de clase media-alta, en los barrios marginales el riesgo se incrementa un $460 \%$. Diferenciando por tipo de violencia, el peso de la situación social es máximo en el caso de la violencia física, intermedio en el caso de la violencia sexual y mínimo en el caso de la violencia psicológica.

Palabras clave: factores de riesgo; violencia en la pareja; análisis cuantitativo; violencia física; violencia sexual; violencia psicológica 
Abstract. Social exclusion as a risk factor for gender-based intimate partner violence

Based on a structural concept of gender-based intimate partner violence and social exclusion, this paper analyses the risk of experiencing such violence according to different intensities and types of social exclusion. To this end, data from the 2013 Foessa Foundation's Survey on Social Integration and Social Needs was used. The Foessa survey aims to analyse social exclusion in Spain and, in its latest edition, also includes a set of questions aimed at detecting gender-based violence processes by the current or former partner in a standardised manner. The data are drawn from 5,473 face-to-face interviews. Bivariate and multivariate analyses are carried out and different types of violence (physical, sexual and psychological) are distinguished. Taking full integration as a reference, the risk of experiencing gender-based violence increases by $45 \%$ in cases of moderate exclusion and by $82 \%$ in cases of severe exclusion. Distinguishing among types of exclusion, a significant increase is observed in cases of social conflict (460\%) and exclusion from education $(88 \%)$ and healthcare $(57 \%)$, while exclusion from employment, consumption, politics, housing and social isolation are not significant. Moreover, taking upper-middle class neighbourhoods as a reference, in deprived neighbourhoods the risk increases by $460 \%$. Distinguishing among types of violence, the influence of the social situation is at its highest in the case of physical violence, intermediate in the case of sexual violence and minimal in the case of psychological violence.

Keywords: risk factors; intimate partner violence; quantitative analysis; physical violence; sexual violence; psychological violence

\section{Sumario}

\section{Introducción}

2. Un tema de investigación necesario que entraña ciertos riesgos

3. Una revisión de los principales factores de riesgo identificados por la literatura
5. Metodología

6. Resultados y discusión

7. Conclusiones

Referencias bibliográficas

\section{Introducción}

En las últimas décadas, la producción teórica sobre violencia de género en la pareja se ha hecho cada vez más abundante, pero ha sido también muy fragmentada, algo que ha facilitado la permanencia de vacíos en la literatura.

Por un lado, de hecho, hallamos investigaciones que carecen por completo de perspectiva de género. Se trata de estudios que frecuentemente utilizan metodologías de análisis cuantitativas y, por medio de estas, suelen identificar factores de riesgo de victimización (Aldarondo y Castro-Fernández, 2011; Callá, 2008; Chan, 2014; Corradi, 2008; Cunradi et al., 2011, 2008; Daoud et al., 2013; González-Guarda et al., 2013; Mavrikiou et al., 2014; Nagassar et al., 2010; Panchanadeswaran et al., 2010; Raj y Silverman, 2002; Renner y Whitney, 2012; Smith et al., 2011; Van Vijk y de Bruijn, 2012). Su mayor limitación reside en el hecho de que, cuando intentan dar cuenta de los mecanismos subyacentes a tales resultados, no tienen en absoluto en cuenta las des- 
igualdades de género que están operando. Es decir, ignoran que si los factores de riesgo pueden actuar como lo hacen es porque se insertan en una estructura social determinada, en la que los hombres disfrutan de mayor valor y poder y se sitúan en una posición de prominencia social, mientras que las mujeres poseen un estatus devaluado y se encuentran en una posición de subordinación (Bourdieu, 1980). Esto revierte asimismo en la construcción de identidades de género también dicotomizadas y jerarquizadas y que también suponen, por su configuración (potenciamiento de la autoestima, el poder, la fuerza, la agresividad y la independencia entre ellos, frente a la debilidad, la dependencia, la amabilidad, la empatía y el autosacrificio entre ellas) (Bourdieu, 1998; Lagarde y De los Ríos, 2008), un aumento del riesgo de victimización en el caso de las mujeres y de agresión en el de los hombres. Tales investigaciones sobre factores de riesgo, además, han tenido un desarrollo limitado a nivel estatal.

Por otro lado, encontramos investigaciones feministas que ponen el acento en el origen estructural y en el carácter transversal de la violencia de género, utilizan prevalentemente metodologías de análisis cualitativas y tienden a ser reacias a reconocer la existencia de elementos de riesgo (o, dicho de otra forma, las diferencias entre grupos, incluso en el marco de una estructura - de género- homogeneizadora).

Como resultado, tanto las unas como las otras solo pueden ofrecer explicaciones parciales. Las primeras, de hecho, no pueden aclarar por qué, si los factores de riesgo afectan tanto a hombres como a mujeres, la propensión a agredir es tan mayoritariamente masculina y los blancos de tales agresiones son tan a menudo mujeres (García y Casado, 2010), mientras que las segundas no pueden dar cuenta de por qué en ciertos grupos (mujeres sin estudios, con discapacidad, migradas, etc.) la incidencia de la violencia de género es mayor (Jewkes et al., 2002).

Precisamente aquí, en el hueco dejado por la intersección entre ambas perspectivas, se sitúa nuestra investigación, en la cual, de hecho, intentamos conjugar el reconocimiento del origen estructural de la violencia de género con un análisis de elementos que pueden incrementar o reducir el riesgo de vivirla.

En este sentido, el trabajo aquí efectuado presenta fuertes puntos de contacto con el marco ecológico integrado de Heise (1998). Existe, sin embargo, una clara diferencia entre los dos: si en aquel las desigualdades de género se consideraban simplemente un elemento causal entre muchos, aquí estas representan la única condición necesaria de la violencia (García y Casado, 2010), y constituyen, por lo tanto, un elemento clave para comprender el fenómeno analizado.

Un segundo elemento a considerar es el hecho de que, aunque los estudios que se ocupan de factores de riesgo son muy numerosos, hasta donde conocemos casi todos analizan el papel desempeñado por la situación económica, mientras que todavía ninguno se ha ocupado de la situación de exclusión social. Se trata de una ausencia difícilmente justificable, sobre todo si se considera que ya son varias las investigaciones que han puesto de relieve la necesidad de analizar el impacto cumulativo de varios factores y sus interre- 
laciones (Aldarondo y Castro-Fernández, 2011; Campbell et al., 2011), algo que guarda una relación directa con la noción de exclusión. La necesidad de incorporar este nuevo concepto, por otra parte, aparece con claridad aún mayor si se tiene en cuenta que este es mucho más capaz que la noción de pobreza de captar las dinámicas de la desigualdad contemporánea, caracterizada precisamente por la emergencia de otros elementos de desigualdad además del económico (Subirats, 2004).

\section{Un tema de investigación necesario que entraña ciertos riesgos}

La presente investigación, como se ha aclarado, se inserta en un hueco existente en la literatura. La permanencia de este vacío teórico en un contexto de proliferación teórica sobre violencia de género remite al hecho de que el tema aquí tratado podría terminar potenciando explicaciones individualistas de la violencia de género, así como dinámicas de criminalización de la población excluida, algo que evidentemente, desde posiciones progresistas, se desea evitar.

Este peligro, sin embargo, se resuelve manteniendo una mirada estructural. En el caso de la violencia de género, esto significa recordar en todo momento que se trata de un fenómeno que solo puede existir en presencia de una precondición necesaria: un trasfondo estructural de relaciones desiguales de género. En el caso de la exclusión social, mantener una mirada estructural significa reconocer que «el corazón de la problemática de la exclusión no está donde encontramos a los excluidos» (Castel, 1995: 108). Muy al contrario, estas situaciones son el producto de las desigualdades estructurales inherentes al sistema capitalista, ulteriormente intensificadas por efecto de los cambios sociales acaecidos en las últimas décadas (Laparra et al., 2007; Subirats, 2004).

Ello implica que los mayores niveles de violencia de género que se detectan en contextos de exclusión deberán reconducirse automáticamente al nivel de la estructura: estructura de género, por cómo esta construye hombres y mujeres determinados, y estructura de clase, por cómo esta también contribuye a caracterizar los valores, las actitudes y los comportamientos de los individuos.

En relación con esto, las reflexiones más interesantes las han efectuado los estudios que se ocupan de interseccionalidad (Almeida y Lockard, 2005; Bograd, 2005; Collins, 1990-2000; Damant et al., 2008; Gandarias, 2016; Nixon y Humphreys, 2010; Prins, 2006; Richie, 2000; Sokoloff, 2004; Sokoloff y Dupont, 2005; Warner y Shields, 2013). Estos, de hecho, parten precisamente de la constatación de que los diferentes sistemas de opresión están interconectados (Collins, 1990-2000) y subrayan que la posición de los individuos solo puede comprenderse a partir de su posicionamiento en diferentes estructuras de opresión que se interseccionan. Por ello estos estudios constituyen un marco de referencia obligado y privilegiado para nuestra investigación.

Los análisis sobre interseccionalidad, sin embargo, tienen carácter casi exclusivamente teórico (Damant et al., 2008) y, además, aun habiendo a menudo subrayado la importancia de considerar una multiplicidad de factores, en la práctica se han ocupado de forma casi exclusiva de la intersección entre género 
y raza o etnia (Choo y Ferree, 2010; Kwan, 1997; Sokoloff y Dupont, 2005). Esto significa que es especialmente necesario tanto efectuar estudios empiricos que se inserten bajo este paradigma como investigar el papel de otras intersecciones. También en esto, entonces, nuestro estudio es novedoso.

\section{Una revisión de los principales factores de riesgo identificados por la literatura}

Como ya se ha apuntado, existen (sobre todo en el ámbito anglosajón) innumerables estudios sobre factores de riesgo. Pese a la frecuente ausencia de perspectiva de género, estos constituyen un referente privilegiado para la presente investigación, ya que son los únicos que ofrecen una información empírica detallada de la interrelación entre violencia de género y posición socioeconómica. En otras palabras, frente a la ausencia de exámenes que se ocupen expresamente de la relación entre violencia de género y exclusión social, estos estudios se configuran como el referente más cercano a nuestro objeto de análisis.

La literatura sobre factores de riesgo es muy extensa y ha analizado la interrelación de la violencia de género con un gran abanico de variables. Entre todas ellas, prestamos especial atención a aquellas que guardan una relación más estrecha con los procesos de exclusión social.

Empezando por el nivel educativo, varios estudios muestran una relación entre este y la violencia de género, tanto en el caso de las mujeres como de los hombres. Con respecto a las primeras, la mayoría de las investigaciones evidencian que un nivel formativo elevado se configura como un elemento de protección, y la falta de estudios, como un factor de riesgo (Campbell et al., 2011; Friedemann-Sánchez y Lovatón, 2012; Khalifeh et al., 2013; Ruiz-Pérez et al., 2006; Van Wijk y de Bruijn, 2012; Vives-Cases et al., 2009, 2010). En el caso de los varones, la existencia de una relación inversa entre logros educativos y violencia de género se confirma (por lo menos en el caso de la violencia física), aunque en este caso lo que se incrementa no es el riesgo de victimización, sino de agresión (Stith et al., 2004; Yick, 2001).

Numerosas investigaciones muestran también la importancia de considerar la esfera laboral y ponen de manifiesto que el desempleo masculino es un claro factor de riesgo de agresión (González y Santana, 2001; Stith et al., 2004; Tauchen y Witte, 2001). Otras (Kiss et al., 2012), sin embargo, no encuentran asociación alguna entre la situación laboral del varón y el riesgo de victimización para la mujer. En lo que respecta al (des)empleo femenino, las evidencias empíricas son discordes (Friedemann-Sánchez y Lovatón, 2012; Stith et al., 2004; Van Wijk y de Bruijn, 2012; Walby y Allen, 2004).

En el caso de la pobreza, cabe diferenciar entre la realidad de los individuos y la del entorno. En lo que atañe a la primera, numerosas investigaciones asocian la falta de recursos económicos con una mayor incidencia de la violencia de género (Jewkes, Levin y Penn-Kekana, 2002; Friedemann-Sánchez y Lovatón, 2012; Khalifeh et al., 2013; Mavrikiou et al., 2014; Ruiz-Pérez et al., 2006; Walby y Allen, 2004), mientras que otras indican que no hay relación 
Tabla 1. Investigaciones internacionales que documentan la existencia de varios factores de riesgo y que están basadas en muestras representativas a nivel estatal o regional

\begin{tabular}{|c|c|c|c|}
\hline & Principales hallazgos & Autoras y autores & País \\
\hline \multirow[t]{2}{*}{$\begin{array}{l}\text { Nivel } \\
\text { educativo }\end{array}$} & $\begin{array}{l}\text { En las mujeres, un nivel formativo elevado es un elemento } \\
\text { de protección, y la falta de estudios, un factor de riesgo. }\end{array}$ & $\begin{array}{l}\text { Campbell et al., 2011; } \\
\text { Van Wijk y de Bruijn, 2012; Vives- } \\
\text { Cases et al., 2009, } 2010\end{array}$ & $\begin{array}{l}\text { EE. UU.; } \\
\text { Curazao; } \\
\text { España }\end{array}$ \\
\hline & $\begin{array}{l}\text { El hecho de que la mujer tenga estudios primarios o inferiores } \\
\text { incrementa la probabilidad de experimentar violencia sexual, } \\
\text { pero no física o psicológica. }\end{array}$ & Ruiz-Pérez et al., 2006 & España \\
\hline
\end{tabular}

El hecho de que la mujer tenga estudios primarios o inferiores
incrementa la probabilidad de experimentar violencia física, pero
no psicológica.

Khalifeh et al., 2013

Inglaterra no psicológica.

\begin{tabular}{lll}
\hline $\begin{array}{l}\text { Aunque la relación es más intensa en el caso de la violencia física, } \\
\text { permanece también en el caso de la psicológica. }\end{array}$ & 2012 & Colombia \\
\hline $\begin{array}{l}\text { No hay relación entre nivel educativo de la mujer y riesgo } \\
\text { de enfrentar violencia de género. }\end{array}$ & $\begin{array}{l}\text { Barrett et al., 2012; Panchana- } \\
\text { deswaran et al., 2010 }\end{array}$ & $\begin{array}{l}\text { Ucrania; } \\
\text { EE. UU. }\end{array}$ \\
\hline $\begin{array}{l}\text { La relación entre nivel educativo y violencia física es más intensa } \\
\text { en el caso de los varones agresores que en el de las mujeres }\end{array}$ & & $\begin{array}{l}\text { Varios } \\
\text { agredidas. }\end{array}$
\end{tabular}

\begin{tabular}{lll}
\hline La relación entre nivel educativo y violencia física (pero & Yick, 2001 & EE. UU.
\end{tabular}
no psicológica) es más intensa en el caso de los varones agresores que en el de las mujeres agredidas.

\begin{tabular}{lll}
\hline $\begin{array}{l}\text { La diferencia de estatus educativo entre los dos miembros de la } \\
\text { pareja no incrementa el riesgo de experimentar violencia de género. }\end{array}$ & Franklin y Menaker, 2014 & EE. UU. \\
$\begin{array}{lll}\text { En el caso de las mujeres, el desempleo es un factor de riesgo, } \\
\text { en cambio, un trabajo regular supone un factor de protección. }\end{array}$ & $\begin{array}{l}\text { Van Wijk y de Bruijn, 2012; } \\
\text { Walby y Allen, 2004 }\end{array}$ & $\begin{array}{l}\text { Curazao; } \\
\text { Reino Unido }\end{array}$ \\
\hline $\begin{array}{l}\text { El desempleo femenino es un factor de riesgo, pero solamente con } \\
\text { respecto a la violencia psicológica y sexual. }\end{array}$ & & España \\
\hline $\begin{array}{l}\text { El efecto protector del empleo femenino es casi insignificante. } \\
\text { El empleo remunerado es un factor de riesgo para la mujer. }\end{array}$ & $\begin{array}{l}\text { Stith et al., 2004 } \\
\text { yriedemann-Sánchez }\end{array}$ & $\begin{array}{l}\text { Varios } \\
\text { (metaanálisis) }\end{array}$ \\
\hline El hecho de que la mujer tenga un empleo remunerado es & Riger y Staggs, 2004 & Colombia \\
\hline
\end{tabular}
un factor de protección cuando el varón también tiene empleo y un factor de riesgo cuando él no lo tiene.

\begin{tabular}{l}
\hline El empleo remunerado es un factor de protección en el caso de $\quad$ Vives-Cases et al., $2010 \quad$ España \\
las mujeres autóctonas y un factor de riesgo entre las mujeres \\
migradas.
\end{tabular}

La relación existente entre paro masculino y violencia de género González y Santana, 2001; Stith España; varios es mucho más intensa que la que se detecta en el caso del et al., 2004; Tauchen y Witte, 2001 (metaanálisis); desempleo femenino.

\begin{tabular}{llc}
\hline $\begin{array}{l}\text { Existe una relación entre desempleo masculino y violencia } \\
\text { de género en área rural pero no en área urbana. }\end{array}$ & Lanier y Maume, 2009 & EE. UU. \\
\hline $\begin{array}{l}\text { No hay asociación entre situación laboral del varón y riesgo } \\
\text { de victimización para la mujer. }\end{array}$ & Kiss et al., 2012 & Brasil
\end{tabular}

El hecho de que ella tenga empleo mientras él está desempleado Riger y Staggs, $2004 \quad$ EE. UU. incrementa el riesgo de victimización.

\begin{tabular}{lll}
\hline El hecho de que ella tenga un sueldo más elevado incrementa & Atkinson et al., 2005; Chung & EE. UU; \\
el riesgo de victimización. & et al., 2008; Fox et al., 2002; & Nicaragua \\
& Grose y Grabe, 2014 & \\
\hline
\end{tabular}


Tabla 1. Investigaciones internacionales que documentan la existencia de varios factores de riesgo y que están basadas en muestras representativas a nivel estatal o regional (continuación)

\begin{tabular}{|c|c|c|c|}
\hline & Principales hallazgos & Autoras y autores & País \\
\hline \multirow[t]{6}{*}{ Pobreza } & $\begin{array}{l}\text { Vivir en un hogar pobre incrementa el riesgo de experimentar } \\
\text { violencia de género. }\end{array}$ & Mavrikiou et al., 2014 & Chipre \\
\hline & $\begin{array}{l}\text { Vivir en un hogar pobre incrementa el riesgo de experimentar } \\
\text { violencia física. }\end{array}$ & Jewkes et al., 2002 & Sudáfrica \\
\hline & $\begin{array}{l}\text { Vivir en un hogar pobre incrementa el riesgo de experimentar } \\
\text { violencia física, pero no psicológica. }\end{array}$ & $\begin{array}{l}\text { Friedemann-Sánchez y Lovatón, } \\
\text { 2012; Khalifeh et al., } 2013\end{array}$ & $\begin{array}{l}\text { Colombia; } \\
\text { Inglaterra }\end{array}$ \\
\hline & $\begin{array}{l}\text { Vivir en un hogar pobre incrementa el riesgo de experimentar } \\
\text { violencia física y sexual, pero no psicológica. }\end{array}$ & Ruiz-Pérez et al., 2006 & España \\
\hline & No hay relación alguna entre pobreza del hogar y violencia. & $\begin{array}{l}\text { Barrett et al., 2012; Kiss et al., } \\
2012\end{array}$ & Ucrania; Brasil \\
\hline & $\begin{array}{l}\text { Residir en un barrio empobrecido incrementa el riesgo de } \\
\text { experimentar violencia de género. }\end{array}$ & $\begin{array}{l}\text { Cunradi et al., 2000; Khalifeh et } \\
\text { al., } 2013\end{array}$ & $\begin{array}{l}\text { EE. UU; } \\
\text { Inglaterra }\end{array}$ \\
\hline \multirow[t]{2}{*}{$\begin{array}{l}\text { Etnia y/0 } \\
\text { lugar de } \\
\text { origen }\end{array}$} & $\begin{array}{l}\text { Entre mujeres migradas, el riesgo de experimentnar violencia } \\
\text { de género se incrementa. }\end{array}$ & $\begin{array}{l}\text { Montañés y Moyano, 2006; Raj y } \\
\text { Silverman, 2002; Vives-Cases et } \\
\text { al., } 2010\end{array}$ & España; EE. UU \\
\hline & $\begin{array}{l}\text { Entre mujeres pertenecientes a minorías étnicas, el riesgo } \\
\text { de experimentnar violencia de género se incrementa. }\end{array}$ & $\begin{array}{l}\text { Daoud et al., 2013; Lanier y } \\
\text { Maume, } 2009\end{array}$ & $\begin{array}{l}\text { Canadá; } \\
\text { EE. UU }\end{array}$ \\
\hline \multirow{5}{*}{$\begin{array}{l}\text { Abuso de } \\
\text { alcohol } \\
\text { y otras } \\
\text { drogas }\end{array}$} & $\begin{array}{l}\text { Entre mujeres con problemas de adicciones la prevalencia } \\
\text { de violencia de género es mayor que en el conjunto de la } \\
\text { población. }\end{array}$ & $\begin{array}{l}\text { Campbell et al., 2011; Kiss et } \\
\text { al., } 2012\end{array}$ & EE. UU.; Brasil \\
\hline & $\begin{array}{l}\text { El hecho de que la mujer tenga problemas de adicciones } \\
\text { no incide en el riesgo de experimentar violencia de género. }\end{array}$ & Stöckl et al., 2011 & Alemania \\
\hline & $\begin{array}{l}\text { El riesgo de experimentar violencia de género es mayor } \\
\text { cuando la pareja masculina consume grandes cantidades } \\
\text { de alcohol. }\end{array}$ & Barrett et al., 2012 & Ucrania \\
\hline & $\begin{array}{l}\text { El riesgo de experimentar violencia física y sexual es mayor } \\
\text { cuando la pareja masculina consume grandes cantidades } \\
\text { de alcohol. }\end{array}$ & $\begin{array}{l}\text { Kiss et al., 2012; Lanier y } \\
\text { Maume, 2009; Stöckl et al., } \\
2011\end{array}$ & $\begin{array}{l}\text { Brasil; EE. } \\
\text { UU.; Alemania }\end{array}$ \\
\hline & $\begin{array}{l}\text { El riesgo de experimentar violencia psicológica (pero } \\
\text { no física ni sexual) es mayor cuando la pareja masculina } \\
\text { consume grandes cantidades de alcohol. }\end{array}$ & Grose y Grabe, 2014 & Nicaragua \\
\hline $\begin{array}{l}\text { Conflicti- } \\
\text { vidad en } \\
\text { la pareja }\end{array}$ & $\begin{array}{l}\text { El hecho de que haya conflictos en la pareja incrementa el } \\
\text { riesgo de experimentar violencia física. }\end{array}$ & $\begin{array}{l}\text { Aldarondoet al., 2002; Heise, } \\
\text { 1998; Jewkes et al., 2002; } \\
\text { Jewkes, } 2002\end{array}$ & $\begin{array}{l}\text { EE.UU.; Sud- } \\
\text { áfrica; varios } \\
\text { (metaanálisis) }\end{array}$ \\
\hline $\begin{array}{l}\text { Discapa- } \\
\text { cidad }\end{array}$ & $\begin{array}{l}\text { El hecho de padecer una discapacidad incrementa el riesgo } \\
\text { de experimentar violencia de género. }\end{array}$ & $\begin{array}{l}\text { Campbell et al., 2011; Smith, } \\
\text { 2008; Stöckl et al., } 2011\end{array}$ & $\begin{array}{l}\text { EE. UU; } \\
\text { Alemania }\end{array}$ \\
\hline
\end{tabular}

alguna entre pobreza y violencia (Barrett et al., 2012; Kiss et al., 2012). En lo que respecta al entorno más amplio, varias investigaciones muestran que residir en un barrio empobrecido incrementa el riesgo de experimentar violencia de género (Cunradi et al., 2000; Khalifeh et al., 2013) y que este efecto se mantiene incluso cuando se controla por el nivel económico del hogar (Khalifeh et al., 2013). 
En lo que respecta a la etnia y/o al lugar de origen, varios estudios detectan una mayor incidencia de la violencia de género en mujeres migradas (Montañés y Moyano, 2006; Raj y Silverman, 2002; Vives-Cases et al., 2010) o pertenecientes a minorías étnicas (Daoud et al., 2013; Lanier y Maume, 2009).

Otro factor de riesgo es el consumo de alcohol u otras drogas por parte de uno de los dos miembros de la pareja. En lo que respecta a las mujeres, los datos disponibles son contradictorios (Campbell et al., 2011; Stöckl et al., 2011; Kiss et al., 2012). En el caso de los varones, por el contrario, la existencia de una relación entre consumo de alcohol y violencia se establece sin lugar a dudas (Barrett et al., 2012; Grose y Grabe, 2014; Kiss et al., 2012; Lanier y Maume, 2009; Stöckl et al., 2011).

Asimismo, varias investigaciones (Aldarondo et al., 2002; Jewkes, 2002; Jewkes et al., 2002) muestran que la presencia de conflictividad en la pareja se asocia a un incremento del riesgo de victimización.

Finalmente, de acuerdo con la literatura existente, otro factor de riesgo muy relevante (Barrett et al., 2012; Jewkes et al., 2002; Kiss et al., 2012; Smith et al., 2011; Stöckl et al., 2011; Van Wijk y de Bruijn, 2012) es la victimización previa, incluyendo en ella tanto las experiencias de violencia de género vividas o presenciadas en momentos anteriores como la violencia de cualquier tipo experimentada en la infancia o en otras etapas. Este elemento, sin embargo, no se analiza aquí porque no guarda una relación directa con procesos de exclusión y es, además, una información que la fuente empleada no recoge.

Por último, cabe señalar que la inexistencia de investigaciones con carácter longitudinal impide establecer con seguridad la direccionalidad de la relación existente entre factores de riesgo y vivencia de la violencia (Barrett et al., 2012; Campbell et al., 2011; Friedemann-Sánchez y Lovatón, 2012; Stöckl et al., 2011). La mayoría de las investigaciones consultadas, sin embargo, considera que, en términos generales, esta puede discurrir en ambos sentidos (Barrett et al., 2012; Josephson, 2005; Khalifeh et al., 2013; Stöckl et al., 2011; Walby y Allen, 2004). Es decir, que el desempleo, la pobreza, las adicciones, etc. pueden ser tanto preexistentes a la violencia como algo que aparece a consecuencia de esta.

Ninguno de los estudios sobre factores de riesgo consultados se ocupa específicamente de la noción de exclusión social, noción que hace referencia a un fenómeno de origen estructural (Laparra et al., 2007; Laparra, 2010; Subirats, 2005), de naturaleza procesual (Boon y Farnsworth, 2011; Oxoby, 2009) y con carácter multidimensional (Boon y Farnsworth, 2011; Laparra et al., 2007; Pirani, 2011; Subirats, 2005; Van Bergen et al., 2014), y que desde su formulación ha cosechado un éxito abrumador, llegando prácticamente a sustituir la idea de pobreza como noción básica en los estudios sobre desigualdad.

Es por ello que, una vez examinado lo que tales estudios descubren, ampliamos la mirada a diferentes ámbitos y disciplinas para intentar comprender cómo una situación específica de exclusión social preexistente puede favorecer el desencadenamiento de violencia de género.

En lo que respecta a las mujeres, algunos estudios evidencian que una situación de dificultad social reduce su capacidad de responder con eficacia a 
la violencia (Hamby, 2005), algo que puede favorecer el recurso a la misma por parte del varón. Otras vinculan situación socioeconómica y autoestima (Falci, 2011; Santo et al., 2013; Veselska et al., 2010; Zhang y Postiglione, 2001), lo cual permite hipotetizar que la relación entre exclusión y violencia se encuentra mediada por esta. Un tercer grupo parte de la constatación de que en las capas más bajas de la sociedad se registra una mayor adhesión a roles de género tradicionales (Elley, 2011), algo que es, de por sí, un factor de riesgo de violencia de género (Villavicencio, 1993). Una última hipótesis, finalmente, sugiere que si las mujeres en situación de exclusión se convierten con más facilidad en blancos de la violencia es porque, como sociedad, las hacemos más vulnerables a la misma.

En el caso de los varones, una teoría ya clásica aclara que las situaciones de estrés y frustración — posiblemente más frecuentes en contextos de dificultad social - pueden actuar como desencadenantes de la agresión (Berkowitz, 1989; Dollard et al., 1939) ${ }^{1}$. Otras teorías hacen hincapié en la violencia estructural como causante de violencia directa, algo que se resume en el famoso axioma de Galtung «la violencia genera violencia» (Galtung, 1990: 266). El hecho de que las mujeres, pese a vivir la misma opresión, apenas recurran a violencia directa, sin embargo, vuelve a poner sobre la mesa el sistema de género. Otras tantas centran su mirada en el conflicto, sea porque este es una fuente de estrés, sea porque la violencia es una táctica frecuentemente usada dentro del mismo (Jewkes, 2002; Walby y Allen, 2004), sobre todo cuando no se dispone de herramientas para manejarlo de otra manera (FriedemannSánchez y Lovatón, 2012). Un cuarto grupo parte de la constatación de que el dominio puede asegurarse de múltiples maneras y aclara que cuanto menor es la capacidad de lograrlo de forma "legítima» (algo que puede asociarse con procesos de exclusión), más probable será el recurso a herramientas ilegítimas, como la violencia (Basile et al., 2013; Callá, 2008; Izquierdo, 2007; Lawson, 2012; Merton, 1968; Walby y Allen, 2004). Un quinto grupo pone el acento en el hecho de que, en las situaciones de dificultad social, puede originarse una definición alternativa de masculinidad en la que la violencia adquiere un papel particularmente relevante (DeKeseredy y Schwartz, 2005; Fodor, 2006; Friedemann-Sánchez y Lovatón, 2012; Hochschild, 1991; Jewkes, 2002; Messerschmidt, 1993; Strier et al., 2014). Otras investigaciones, finalmente, ponen de relieve los mayores niveles de desigualdad de género que caracterizarían a los colectivos excluidos (Kiss et al., 2012; Smith, 1990; Uthman et al., 2009).

En cualquier caso, es importante resaltar que todos estos elementos constituyen factores de riesgo, pero no factores causales. Es decir que si operan como lo hacen es únicamente porque actúan en presencia de una precondición

1. Otros autores y autoras, sin embargo, matizan este concepto y explican que tanto la probabilidad de que dicha agresión tenga lugar (Bandura, 1979) como la dirección que esta tome (Hochschild, 2009) guardan una relación directa con las consecuencias anticipadas de la misma, y esta es una aportación fundamental para comprender los procesos de violencia de género y, más concretamente, la razón por la cual la violencia masculina se dirige preferentemente contra la pareja femenina. 
necesaria y a ellos externa: un trasfondo estructural de relaciones desiguales de género (Jewkes, 2002; Jewkes et al., 2002). De lo contrario, no se podría comprender por qué, si tales factores inciden tanto en hombres como en mujeres, provocan un incremento de la agresión masculina sobre la pareja femenina, pero no lo contrario.

\section{Metodología}

Con este estudio se quiere cuantificar el riesgo de vivir violencia de género en la pareja en las situaciones de integración y en los distintos espacios y dimensiones de la exclusión social. Este objetivo general se traduce en dos objetivos específicos:

1. Cuantificar el riesgo de experimentar violencia de género en función de diferentes intensidades, dimensiones e indicadores de exclusión.

2. Cuantificar el riesgo de experimentar diferentes tipos de violencia de género (física, sexual y psicológica) en función de diferentes niveles de intensidad de la exclusión.

Las hipótesis de las que se parte son:

1. La violencia de género es un fenómeno con causas estructurales que afecta a mujeres de toda condición social.

2. En situaciones de exclusión la incidencia de la violencia de género es mayor.

3. Al intensificarse la exclusión también aumentará el riesgo de experimentar violencia.

4. El incremento variará en función del tipo de exclusión.

5. El incremento será especialmente pronunciado en el caso de la violencia física.

\subsection{La Encuesta de la Fundación Foessa sobre Integración Social y Necesidades Sociales de 2013}

Este estudio utiliza datos extraídos de la Encuesta de la Fundación Foessa sobre Integración Social y Necesidades Sociales de 2013. Se trata de la tercera de una serie de encuestas realizadas en 2007, 2009 y 2013, cuyo objetivo es cuantificar y analizar las condiciones de vida y la exclusión social en el Estado español, obteniendo datos representativos a nivel nacional y —en esta última edición - también de trece comunidades autónomas. El universo está formado por la totalidad de hogares del Estado español, con la excepción de Ceuta y Melilla. El trabajo de campo se realizó entre abril y agosto de 2013, cuando se efectuaron encuestas presenciales a 8.776 hogares (que han aportado información sobre 24.776 personas). De estas, 5.473 encuestas fueron contestadas por mujeres y han podido ser utilizadas para la presente investigación.

La encuesta contiene dos cuestionarios, uno de filtro y uno principal, con la mayor parte de las preguntas. Este último se realiza únicamente a las personas 
que cumplen determinadas condiciones del primer cuestionario y a una parte de las que no las cumplen. Este procedimiento garantiza que se obtenga un número suficiente de hogares en situación de exclusión. El procedimiento de selección de los hogares es bietápico (se ha efectuado una primera selección de secciones censales y una segunda mediante rutas aleatorias). La selección de dichas secciones no ha sido proporcional al conjunto de la población, sino a la población con indicios de exclusión estimada para la Encuesta de 2007 y a su vez basada en el Censo de Población y Viviendas de 2001. Dicha desproporción en la selección se ha corregido a posteriori mediante ponderación.

La Encuesta de 2013 incluye también preguntas destinadas a detectar procesos de violencia de género de forma estandarizada (trámite de ítems que describen hechos concretos constitutivos de violencia), algo que no encontramos en las anteriores. Se alinea, por lo tanto, con el procedimiento utilizado por otras encuestas expresamente diseñadas para captar tales procesos (macroencuestas de violencia de género 1999, 2002, 2006 y 2011 y Macroencuesta de Violencia contra las Mujeres 2015).

La decisión de recurrir a esta encuesta en lugar de basarse en aquellas responde a la riqueza de la información que esta recoge acerca de los procesos de exclusión. Las macroencuestas, de hecho, apenas aportan datos acerca del estatus socioeconómico de las mujeres y sus parejas, mientras que la información que esta recoge acerca de los procesos de exclusión es amplia y pormenorizada. Ello permite realizar un análisis multidimensional, distinguir las diferentes dimensiones que conforman los procesos de exclusión, medir la intensidad de los mismos, etc.

Otra ventaja de esta encuesta con respecto a las que van explícitamente destinadas a captar procesos de violencia de género es el procedimiento de muestreo, expresamente pensado para garantizar la presencia de un número suficiente de hogares en situación de exclusión social. Si se considera que la representación insuficiente de sectores minoritarios de la población es un problema grave que afecta a la práctica totalidad de las encuestas sobre violencia de género (Ferrer et al., 2006), se puede comprender la relevancia de este segundo elemento.

Finalmente, el hecho de formular preguntas sobre violencia de género en el marco de una encuesta que se ocupa de pobreza y exclusión puede facilitar que las mujeres reconozcan con más facilidad la violencia vivida, ya que esta puede ser percibida más fácilmente como un elemento más de las situaciones de dificultad social y resultar, por lo tanto, menos estigmatizadora. La mayor incidencia de violencia física arrojada por la Encuesta Foessa en comparación con otras fuentes (Macroencuesta 2011) parece ser precisamente el resultado de un proceso de este tipo.

Una limitación de esta fuente (y de la práctica totalidad de las encuestas que se ocupan de violencia de género), finalmente, es su carácter transversal, que no permite conocer la secuencia temporal de los hechos (Coker et al., 2011). Para ello, en efecto, se necesitarían encuestas de carácter longitudinal, que, sin embargo, en la actualidad no se llevan a cabo en España. 


\subsection{Definición de variables}

Como ya se ha apuntado, las encuestas de la fundación Foessa sobre integración social y necesidades sociales de 2007 y 2009 no permitían detectar situaciones de violencia de género. En 2012, sin embargo, se nos ofreció la posibilidad de incorporar algunas modificaciones al nuevo cuestionario, hecho que facilitó introducir una batería de nueve ítems que permiten detectar este fenómeno de la forma estandarizada que es habitual. Estos ítems, que aparecen esquematizados en la tabla 2, son: «Le insulta o amenaza», «En ciertas ocasiones le produce miedo», «Le dice que todas las cosas que hace están mal, que es torpe», "Le impide ver a su familia o tener relaciones con amigos, vecinos, etc.», "No le deja trabajar o estudiar", "Decide las cosas que ella puede o no hacer», «Le quita el dinero que ella gana o no le da lo suficiente que necesita para mantenerse», «Cuando se enfada llega a empujar o golpear»e «Insiste en tener relaciones sexuales aunque sepa que ella no tiene ganas».

Para escoger tales ítems se tuvo como referencia a los 26 que estaban contemplados por la Macroencuesta de Violencia de Género 2011. Esta decisión se debía tanto al hecho de que la Macroencuesta representaba en ese momento un referente teórico ineludible como al hecho de que mantener las mismas formulaciones favorecería la comparabilidad de las dos fuentes. A partir de allí, para definir la nueva batería, se tuvo en cuenta tanto la gravedad de la situación descrita y la claridad de la formulación de cada ítem como la necesidad de permitir la detección de todos los diferentes tipos de violencia de género. Se mantuvieron, por lo tanto, los dos únicos ítems referidos respectivamente a la violencia física y sexual, y los siete restantes se dividieron entre violencia psicológica emocional —que identifica situaciones de menosprecios, burlas, insultos, intimidaciones y amenazas—; violencia psicológica de control - que se da cuando la pareja controla las amistades o los horarios de la mujer o cuando le impide trabajar o estudiar-, y violencia económica. Como puede observarse en la tabla que aparece a continuación, en términos generales, se consideró que, al tratarse en todos los casos de ítems que identificaban situaciones especialmente graves y claramente definidas, el simple hecho de que uno de ellos se diera, aunque fuera "rara vez», era suficiente para deducir presencia de violencia de género. En el caso específico de la violencia sexual, sin embargo, la formulación presentaba cierto grado de ambigüedad, con lo cual se exigió que el comportamiento que esta describe se diera «frecuentemente»o «a veces» para poder determinar presencia de violencia en la pareja.

Partiendo de dichos parámetros, se construyeron las variables dependientes del análisis:

- Violencia de género. Cuando la mujer entrevistada ha contestado afirmativamente a como mínimo 1 de los 9 ítems incluidos («frecuentemente», «a veces» $\mathrm{o}$ «rara vez» en términos generales; «frecuentemente» $\mathrm{O}$ "a veces» en el caso del ítem referido a la violencia sexual).

- Violencia psicológica. Cuando la mujer entrevistada ha contestado afirmativamente («frecuentemente», «a veces» $\mathrm{o}$ «rara vez») a alguno de los ítems 
Tabla 2. Variables dependientes. Los ítems que permiten identificar situaciones de violencia de género en la Encuesta Foessa sobre Integración Social y Necesidades Sociales 2013

\begin{tabular}{lll}
\hline Tipo de violencia & Frecuencia requerida & Ítem considerado \\
\hline Violencia psicológica & «Frecuentemente», & Le insulta o amenaza. \\
emocional & «a veces» o «rara vez» & En ciertas ocasiones le produce miedo. \\
Violencia psicológica & & Le dice que todas las cosas que hace están mal, \\
de control & que es torpe. \\
Violencia económica & Le impide ver a su familia o tener relaciones con \\
Violencia física & amigos, vecinos, etc. \\
& No le deja trabajar o estudiar. \\
& Decide las cosas que ella puede o no hacer. \\
& & Le quita el dinero que ella gana o no le da lo \\
& suficiente que necesita para mantenerse. \\
& Cuando se enfada llega a empujar o golpear. \\
\hline Violencia sexual & «Frecuentemente» & Insiste en tener relaciones sexuales aunque sepa \\
& o «a veces» & que ella no tiene ganas. \\
\hline
\end{tabular}

relativos a la violencia psicológica, pero no ha experimentado violencia física ni sexual.

- Violencia física. Cuando la mujer entrevistada ha contestado afirmativamente («frecuentemente», «a veces» o «rara vez») al ítem relativo a la violencia física, independientemente de si se da también violencia psicológica o sexual.

- Violencia sexual. Cuando la mujer entrevistada ha contestado afirmativamente ("frecuentemente» $\mathrm{o}$ "a veces») al ítem relativo a la violencia sexual, independientemente de si se da también violencia psicológica o física.

En lo que respecta a la definición operativa de las situaciones de exclusión social, nos hemos remitido a la que se utiliza en los informes Foessa, a su vez plasmada a partir de una definición empírica anteriormente consensuada entre los más importantes investigadores de ámbito estatal (Laparra et al., 2007). Esta propuesta, que puede verse esquematizada en la tabla 4, considera tres ejes fundamentales (económico, político y social-relacional), que se descomponen en ocho dimensiones (exclusión del empleo, del consumo, política, de la vivienda, de la educación y de la salud, aislamiento social y conflicto social), y estas, a su vez, en 35 indicadores que identifican «situaciones fácticas, constatables empíricamente, que suponían cada una de ellas suficiente gravedad como para poner en cuestión la plena participación social de las personas afectadas» (Laparra et al., 2014: 4). Tales indicadores se agregan, luego, en un índice sintético de excusión social (ISES) que se obtiene atribuyendo a cada uno de ellos un peso inverso a su frecuencia ${ }^{2}$ y que sirve también para crear una clasificación en cuatro grupos:

2. Más en detalle: por cada indicador se calcula el inverso de la frecuencia en 2007 (1/f); luego, en cada dimensión (participación de la producción, del producto social, de los 
- Integración plena: ISES = 0

- Integración precaria: $0<$ ISES $<2$

- Exclusión moderada: $2<$ ISES $<4$

- Excusión severa: ISES $\geq 4$

El indicador número 28 ( Alguien en el hogar ha recibido o recibe malos tratos físicos o psicológicos en los últimos 10 años»), sin embargo, presentaba una clara asociación con situaciones de violencia de género, algo que se ve confirmado por el coeficiente de correlación existente entre estas dos variables, que alcanza unos valores $(0,369)$ mucho más elevados que los de cualquier otro indicador. Para evitar que esto pudiera sesgar nuestro análisis, hemos modificado la batería eliminando el indicador problemático. El ISES también se ha vuelto a calcular.

\subsection{Técnicas estadísticas utilizadas}

En todos los casos, para orientar el análisis de regresión nos hemos remitido al modelo propuesto por Stöckl et al. (2011), inspirado en el marco ecológico formulado precisamente por Heise en 1998. Siguiendo la propuesta de estas autoras, hemos diferenciado entre factores de riesgo relativos a diferentes niveles de análisis (el individuo, el hogar, la relación de pareja y el entorno más amplio) y además hemos añadido otro nivel que hace referencia a la situación de exclusión social. Si hemos añadido este último nivel en lugar de englobar la noción de exclusión en alguno de los otros, es porque se trata de un concepto complejo que reúne en sí indicadores que hacen referencia a niveles diferentes del marco ecológico, desde el individual (IND 10) hasta el hogar (IND 14-21) y el entorno (IND 19).

Para cada uno de los factores de riesgo hemos realizado análisis descriptivos y calculado el chi cuadrado. En un segundo momento, hemos aplicado a cada uno de ellos análisis de regresión logística y hemos estimado los crude odds ratios (OR). Todos los factores que, con este procedimiento, han resultado ser significativos $(p<0,005)$ han sido introducidos en varios modelos de regresión logística multivariante que incluían todas las variables pertenecientes a un mismo nivel del marco ecológico. De esta manera hemos podido observar cuáles eran las variables significativas en cada nivel, una vez controladas las demás variables del mismo. Finalmente, los factores que seguían siendo significativos en estos modelos intermedios se han introducido en el modelo final. $\mathrm{Si}$, en este proceso, alguna variable pierde significación, se crea un modelo de resumen que incluye solamente las variables significativas.

derechos políticos, de la educación, de la vivienda, de la salud, del conflicto social y del aislamiento social), estas cifras se suman y el resultado se divide por el número de indicadores de cada dimensión $(1 / f 1+1 / f 2 \ldots) / N$. Finalmente, los resultados así obtenidos se normalizan para que su suma sea 1 . El valor así obtenido es el peso de cada indicador (Laparra et al., 2014). 
La utilización de un esquema inspirado en el marco ecológico resulta tanto más relevante en cuanto, pese al creciente éxito de esta formulación, la práctica totalidad de la producción científica que se engloba bajo este planteamiento se ha concretado en estudios de carácter teórico, mientras que apenas hay investigaciones de carácter empírico (De Alencar-Rodrígues y Cantera, 2012).

Siguiendo este planteamiento, ante todo se presentan tres modelos de regresión referidos al conjunto de la violencia de género. Estos miden el riesgo de victimización en función de la intensidad, las dimensiones y los indicadores de exclusión contemplados. En un segundo momento se presentan, además, otros tres modelos, que miden el riesgo de experimentar cada tipo de violencia (física, sexual y psicológica) en función de las diferentes intensidades de la exclusión.

Los modelos que miden el riesgo de experimentar violencia en función de la intensidad de la exclusión incluyen (además de la variable exclusión): estado civil, discapacidad, creencias religiosas e ideología para el nivel individual; presencia de algún/a menor o persona mayor y tamaño del hogar para el nivel de hogar; zona de residencia, riqueza provincial, tamaño del lugar de residencia y estado del barrio para el nivel de entorno.

En el modelo que mide el riesgo en función de las distintas dimensiones que conforman la exclusión, además de las variables anteriores, se contempla también el origen, la edad, la relación con la actividad laboral y la situación económica del hogar; mientras que en el modelo que mide el riesgo en función de distintos indicadores de exclusión, se ha añadido el origen, el nivel educativo, la relación con la actividad laboral y la situación económica del hogar. Estas variables se han podido incluir aquí, pero no en el primer modelo, porque se utilizan para crear la variable exclusión, pero no para construir las dimensiones ni los indicadores de exclusión aquí incorporados.

\section{Resultados y discusión}

Como puede observarse en el gráfico 1, según la Encuesta Foessa 2013, en el momento en que se realizó, el $4,1 \%$ de las mujeres experimentaba violencia física; el 4,2\%, violencia sexual, y el 3,1\%, violencia (exclusivamente) psicológica. Si se comparan estos resultados con los obtenidos a partir de los mismos ítems en la Macroencuesta 2011, se observan algunas diferencias: en esta última la prevalencia de violencia física y sexual era menor (se situaba en el $1,6 \%$ y el $3,4 \%$, respectivamente) y —en consecuencia - la presencia de violencia (exclusivamente) psicológica era mayor (la experimentaba el 5,2\% de las mujeres entrevistadas).

Las mayores diferencias, en suma, se registran en el caso de la violencia física. Esto, como ya se ha aclarado en el apartado metodológico, remite al diferente marco en el que se realizaron las preguntas en un caso y en otro.

Observamos ahora cómo varía el riesgo de experimentar violencia de género en función de diferentes intensidades y tipos de exclusión, distinguiendo entre el conjunto de la violencia de género y las realidades específicas de la violencia física, sexual y psicológica. 
Gráfico 1. Porcentaje de mujeres que sufren violencia física, sexual y psicológica según la Encuesta Foessa 2013 y la Macroencuesta 2011

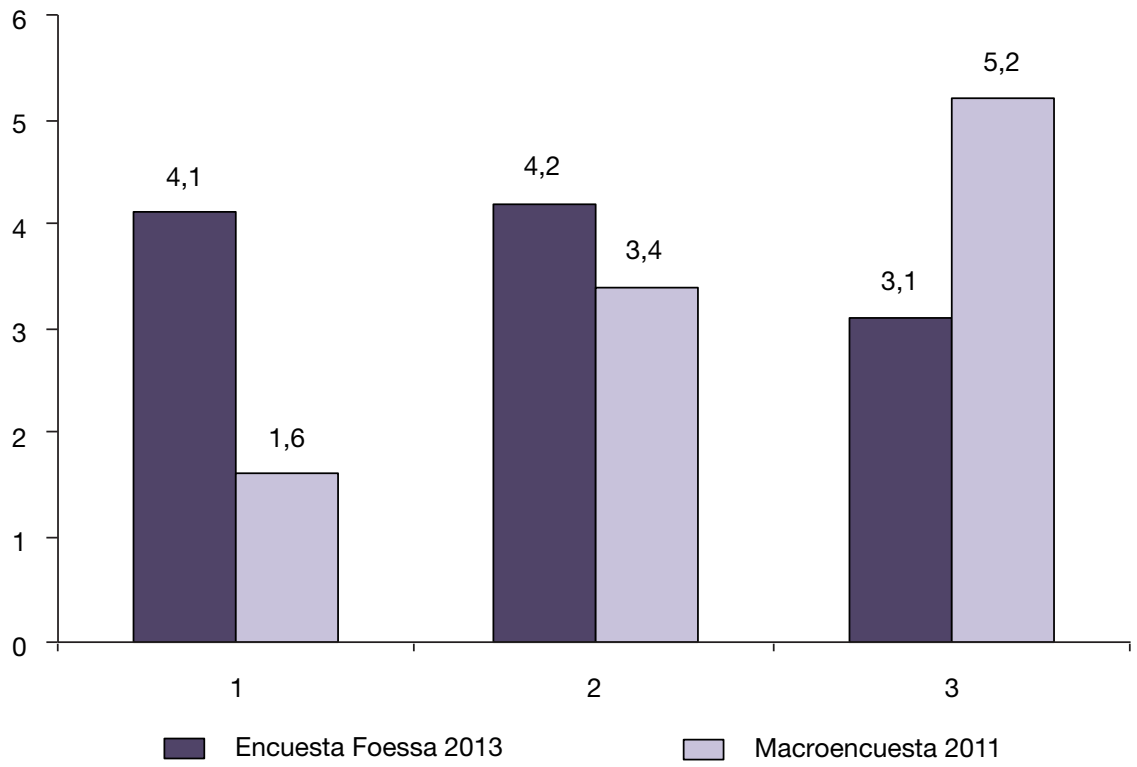

Fuente: Encuesta Foessa sobre Integración Social y Necesidades Sociales 2013 y Macroencuesta de Violencia de Género 2011.

\subsection{El conjunto de la violencia de género}

Los datos descriptivos, que aparecen reflejados en el gráfico 2, muestran que, conforme aumenta la intensidad de la exclusión, también crece el porcentaje de mujeres que sufre violencia de género por parte de la pareja o de la expareja, tanto que en exclusión severa este es 2,5 veces más elevado que en integración plena.

El Índice Sintético de Exclusión Social (ISES), por su parte, también confirma la existencia de una correlación entre las situaciones de violencia de género y los procesos de exclusión social. Entre las mujeres que experimentan violencia de género, de hecho, el ISES es, de media, dos veces más elevado que entre aquellas que no la sufren $(2,40$ frente a 1,21$)$.

Si analizamos la incidencia de la violencia de género en las distintas dimensiones de la exclusión, descubrimos que el riesgo de sufrir maltrato por parte de la pareja o la expareja varía de forma muy clara en función de la dimensión considerada. Tal y como puede observarse en la tabla 3, de hecho, este:

- Se dispara en el caso de mujeres que experimentan una situación de conflicto social (un 38,4\%).

- Es menos elevado pero igualmente significativo en el caso de mujeres que vivencian exclusión de la educación (un 16,2\%), de la salud (un 
Gráfico 2. Porcentaje de mujeres en situación de integración plena, integración precaria, exclusión moderada y exclusión severa que, en la actualidad, experimenta violencia de género por parte de la pareja o de la expareja

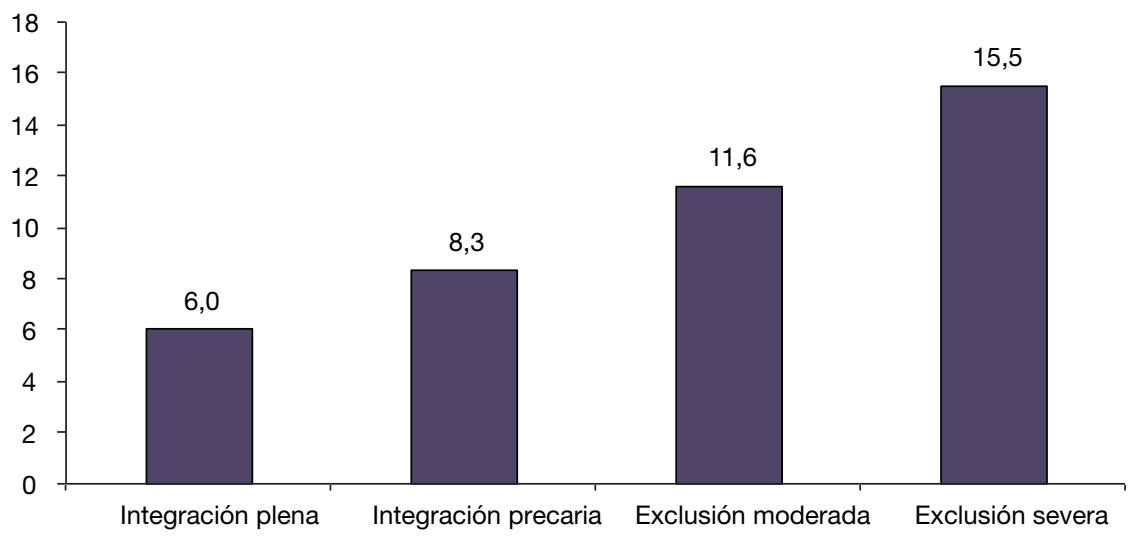

Fuente: Encuesta Foessa sobre Integración Social y Necesidades Sociales 2013.

Tabla 3. Porcentaje de mujeres que, en la actualidad, experimenta violencia de género por parte de la pareja o de la expareja sobre el total de mujeres que viven en hogares afectados por cada dimensión de exclusión

\begin{tabular}{lcc}
\hline & Violencia de género & Incidencia de la dimensión \\
\hline Exclusión del empleo & $9,9^{* \star}$ & 33,7 \\
Exclusión del consumo & 10,1 & 6,1 \\
Exclusión política & $10,8^{*}$ & 11,8 \\
Exclusión de la educación & $16,2^{* \star *}$ & 8,6 \\
Exclusión de la vivienda & $12,0^{* * *}$ & 26,1 \\
Exclusión de la salud & $14,6^{* * *}$ & 19,4 \\
Aislamiento social & $7,8^{* \star *}$ & 5,6 \\
Conflicto social & $38,4^{* \star}$ & 3,8 \\
Total & 8,4 & 100,0 \\
\hline
\end{tabular}

${ }^{*} p<0,050 ;{ }^{* *} p<0,010 ;{ }^{* * *} p<0,001$

Fuente: Encuesta Foessa sobre Integración Social y Necesidades Sociales 2013.

$14,6 \%$ ), de la vivienda (un $12,0 \%$ ), de la política (un $10,8 \%$ ) y del empleo (un 9,9\%).

- No varía de forma significativa en el caso de la exclusión del consumo y en situaciones de aislamiento social.

El hecho de que una situación de aislamiento social no se asocie con un mayor riesgo de victimización es algo manifiestamente contraintuitivo. Un análisis por indicadores (tabla 4), sin embargo, ayuda a comprender este resultado: 
Tabla 4. Porcentaje de mujeres que, en la actualidad, experimenta violencia de género por parte de la pareja o de la expareja sobre el total de mujeres que viven en hogares afectados por cada indicador de exclusión

\begin{tabular}{|c|c|c|c|c|c|}
\hline Eje & Dimensión & & Indicadores Foessa & $\mathrm{N}$ & $\begin{array}{c}\text { Violencia } \\
\text { de género } \\
(\%)\end{array}$ \\
\hline \multirow[t]{8}{*}{ Económico } & Empleo & & Sustentador principal en paro desde hace un año o más. & 502 & 8,8 \\
\hline & & & Sustentador principal con un empleo de exclusión (por el tipo de empleo). & 179 & 10,4 \\
\hline & & & $\begin{array}{l}\text { Sustentador principal con un empleo de exclusión (sin cobertura de la } \\
\text { Seguridad Social). }\end{array}$ & 143 & 11,9 \\
\hline & & & $\begin{array}{l}\text { Hogares sin personas ocupadas, de baja, con prestaciones contributivas } \\
\text { por desempleo o pensiones contributivas. }\end{array}$ & 643 & $13,3^{\star *}$ \\
\hline & & & $\begin{array}{l}\text { Hogares con personas en paro y sin haber recibido formación ocupacional } \\
\text { en el último año. }\end{array}$ & 1576 & 8,8 \\
\hline & & & Todas las personas activas en paro. & 1042 & 11,0 * \\
\hline & Ingresos & & Pobreza extrema (menos de $3.000 € /$ año). & 349 & 11,3 \\
\hline & Privación & & $\begin{array}{l}\text { Hogares que no cuentan con algún bien considerado básico por más del } \\
95 \% \text { de la sociedad por no poder permitírselo. }\end{array}$ & 124 & 6,0 \\
\hline \multirow[t]{19}{*}{ Político } & $\begin{array}{l}\text { Participación } \\
\text { política }\end{array}$ & & $\begin{array}{l}\text { Hogares con alguna persona mayor de edad y de nacionalidad extra- } \\
\text { comunitaria. }\end{array}$ & 263 & 11,4 \\
\hline & & & $\begin{array}{l}\text { No participan en las elecciones por falta de interés y no son miem- } \\
\text { bros de ninguna entidad ciudadana. }\end{array}$ & 464 & 10,0 \\
\hline & Educación & 11 & Hogares con menores de 3 a 15 años no escolarizados. & 72 & 20,0 ** \\
\hline & & 12 & Hogares en los que nadie de 16 a 64 años tiene estudios. & 283 & $14,2^{*}$ \\
\hline & & & $\begin{array}{l}\text { Hogares con alguna persona de } 65 \text { o más años que no sabe leer ni } \\
\text { escribir. }\end{array}$ & 304 & $17,0^{\star \star \star}$ \\
\hline & Vivienda & 14 & Infravivienda. & 38 & 3,2 \\
\hline & & 15 & Deficiencias graves en la construcción, ruina, etc. & 74 & 2,2 \\
\hline & & 16 & Humedades, suciedad y olores (insalubridad). & 514 & 13,1 ** \\
\hline & & 17 & Hacinamiento grave $\left(<15 \mathrm{~m}^{2} /\right.$ persona $)$. & 255 & $13,6^{*}$ \\
\hline & & 18 & Tenencia en precario. & 94 & 10,0 \\
\hline & & 19 & Entorno degradado. & 92 & 17,0 ** \\
\hline & & 20 & $\begin{array}{l}\text { Barreras arquitectónicas con personas con discapacidad física en el } \\
\text { hogar. }\end{array}$ & 286 & 10,5 \\
\hline & & & $\begin{array}{l}\text { Gastos excesivos de la vivienda (ingresos - gastos viv }<\text { umbral } \\
\text { pobreza extrema). }\end{array}$ & 628 & $12,7^{* *}$ \\
\hline & Salud & 22 & Alguien sin cobertura sanitaria. & 14 & 7,1 \\
\hline & & 23 & $\begin{array}{l}\text { Han pasado hambre en los } 10 \text { últimos años con frecuencia o la están } \\
\text { pasado ahora. }\end{array}$ & 259 & $24,5^{\star \star \star}$ \\
\hline & & & $\begin{array}{l}\text { Todos las personas adultas con discapacidad o enfermedades que } \\
\text { generan limitaciones para las actividades cotidianas. }\end{array}$ & 168 & 3,5 \\
\hline & & 25 & Hogares con personas dependientes sin ayuda. & 85 & 11,1 \\
\hline & & & $\begin{array}{l}\text { Hogares con personas enfermas que no han usado los servicios sani- } \\
\text { tarios en un año. }\end{array}$ & 66 & 28,9 *** \\
\hline & & & $\begin{array}{l}\text { Hogares que han dejado de comprar medicinas, seguir tratamientos } \\
\text { o dietas por problemas económicos. }\end{array}$ & 863 & $15,5^{\star \star \star}$ \\
\hline
\end{tabular}


Tabla 4. Porcentaje de mujeres que, en la actualidad, experimenta violencia de género por parte de la pareja o de la expareja sobre el total de mujeres que viven en hogares afectados por cada indicador de exclusión (continuación)

\begin{tabular}{|c|c|c|c|c|c|}
\hline Eje & Dimensión & N. & Indicadores Foessa & $\mathrm{N}$ & $\begin{array}{l}\text { Violencia } \\
\text { de género } \\
(\%)\end{array}$ \\
\hline \multirow{7}{*}{$\begin{array}{l}\text { Social } \\
\text { (relacional) }\end{array}$} & \multirow{4}{*}{$\begin{array}{l}\text { Conflicto } \\
\text { social }\end{array}$} & 29 & Hogares con relaciones muy malas, malas o más bien malas. & 45 & $35,7^{* \star *}$ \\
\hline & & 30 & $\begin{array}{l}\text { Alguien tiene o ha tenido en los } 10 \text { últimos años problemas con el alcohol, } \\
\text { otras drogas o el juego. }\end{array}$ & 140 & $46,3^{\star \star \star}$ \\
\hline & & 31 & Alguien ha sido o está a punto de ser madre adolescente sin pareja. & 61 & $25,7^{\star \star \star}$ \\
\hline & & 32 & Alguien tiene antecedentes penales o los ha tenido en los 10 últimos años. & 50 & $41,2^{\star \star *}$ \\
\hline & \multirow[t]{3}{*}{$\begin{array}{l}\text { Aislamiento } \\
\text { social }\end{array}$} & 33 & $\begin{array}{l}\text { Personas sin relaciones en el hogar y que no cuentan con ningún apoyo } \\
\text { para situaciones de enfermedad o dificultad. }\end{array}$ & 266 & $2,7^{*}$ \\
\hline & & 34 & Hogares con malas o muy malas relaciones con los vecinos. & 31 & 18,2 \\
\hline & & 35 & $\begin{array}{l}\text { Hogares con personas en instituciones: hospitales y pisos psiquiátricos, } \\
\text { centros de deshabituación, para menores, para transeúntes, centros peni- } \\
\text { tenciarios y afines. }\end{array}$ & 15 & $60,0^{\star * \star *}$ \\
\hline Total & & & & 5.473 & 8,4 \\
\hline
\end{tabular}

${ }^{*} p<0,050 ;{ }^{* \star} p<0,010 ;{ }^{* \star *} p<0,001$

Fuente: Encuesta Foessa sobre Integración Social y Necesidades Sociales 2013.

muestra que en esta dimensión se incluyen tanto indicadores que incrementan el riesgo de victimización (IND 35) como otros que lo reducen (IND 33). Más en detalle, vivir en hogares con personas en instituciones (centros de deshabituación, para menores, para transeúntes, centros penitenciarios, etc.) parece incrementar muchísimo la probabilidad de experimentar violencia de género, mientras que no tener relaciones en el hogar y no contar con ningún apoyo para situaciones de enfermedad o de dificultad se asocia a una reducción muy significativa de la misma. Este último dato puede resultar sorprendente. Sin embargo, si se considera que se trata de una situación en la que se encuentran mayoritariamente mujeres mayores viudas, se comprende por qué la incidencia de la violencia de género es tan baja.

El análisis descriptivo realizado refleja con toda claridad la existencia de una relación entre violencia de género y exclusión social. Esta relación, sin embargo, podría estar siendo amplificada (o reducida) por efecto de otras variables asociadas a tal situación (estado civil, edad, etc.). Para lograr una mejor comprensión del fenómeno, el estudio de carácter descriptivo realizado debe ser combinado con un análisis de tipo multifactorial.

Este, tal y como puede observarse en la tabla 5, confirma la relevancia de la situación de exclusión ya detectada por medio de las técnicas descriptivas. El peso de este factor, sin embargo, es ahora claramente menor: ya no se registran diferencias significativas entre la situación de integración plena y precaria, la exclusión moderada se asocia a un incremento del $45 \%$ en la probabilidad de experimentar violencia de género y la exclusión severa a un aumento del $82 \%$ (antes, en este caso, el riesgo se multiplicaba por 2,5). 
Tabla 5. Probabilidad de experimentar violencia de género por parte de la pareja o de la expareja. Modelo que incluye la variable de exclusión social globalmente considerada

\begin{tabular}{|c|c|c|c|c|c|c|c|c|c|c|}
\hline & \multicolumn{3}{|c|}{ Paso 1} & \multicolumn{2}{|l|}{ Paso 2} & \multicolumn{2}{|l|}{ Paso 3} & \multicolumn{3}{|c|}{ Paso 4} \\
\hline & OR & $\mathrm{Cl}$ & $p$ valor & OR & $p$ valor & $\mathrm{OR}$ & $p$ valor & OR & $\mathrm{Cl}$ & $p$ valor \\
\hline \multicolumn{11}{|l|}{ Variable de resumen } \\
\hline \multicolumn{11}{|l|}{ Situación de exclusión social } \\
\hline Integración plena (ref.) & & & 0,000 & & 0,001 & & 0,002 & & & 0,017 \\
\hline Integración precaria & 1,419 & $080-1,864)$ & 0,012 & $1,181(0,891-1,564)$ & 0,247 & $1,164(0,878-1,543)$ & 0,290 & 1,078 & $(0,806-1,442)$ & 0,613 \\
\hline Exclusión moderada & 2,071 & $473-2,912)$ & 0,000 & $1,673(1,174-2,382)$ & 0,004 & $1,610(1,128-2,298)$ & 0,009 & 1,446 & $(1,000-2,091)$ & 0,050 \\
\hline Exclusión severa & 2,875 & $953-4,231)$ & 0,000 & $2,126(1,417-3,190)$ & 0,000 & $2,020(1,341-3,042)$ & 0,001 & 1,823 & $(1,182-2,811)$ & 0,007 \\
\hline \multicolumn{11}{|l|}{ Características de la mujer } \\
\hline \multicolumn{11}{|l|}{ Discapacidad } \\
\hline \multicolumn{11}{|l|}{ No (ref.) } \\
\hline Sí & & & & $2,188(1,502-3,186)$ & 0,000 & $2,224(1,527-3,241)$ & 0,000 & 2,034 & $(1,381-2,995)$ & 0,000 \\
\hline \multicolumn{11}{|l|}{ Estado civil } \\
\hline Casada (ref.) & & & & & 0,000 & & 0,000 & & & 0,000 \\
\hline Pareja de hecho & & & & $2,240(1,532-3,274)$ & 0,000 & $2,224(1,520-3,254)$ & 0,000 & 1,944 & $(1,308-2,890)$ & 0,001 \\
\hline Separada/divorciada & & & & $6,212(4,605-8,380)$ & 0,000 & $6,798(4,927-9,378)$ & 0,000 & 6,698 & $(4,794-9,359)$ & 0,000 \\
\hline Viuda & & & & $1,456 \quad(, 841-2,519)$ & 0,180 & $1,752(, 968-3,171)$ & 0,064 & 2,133 & $(1,163-3,914)$ & 0,014 \\
\hline Soltera & & & & $1,814(1,301-2,529)$ & 0,000 & $1,973(1,393-2,796)$ & 0,000 & 1,812 & $(1,265-2,594)$ & 0,001 \\
\hline \multicolumn{11}{|l|}{ Características del hogar } \\
\hline \multicolumn{11}{|l|}{ N. ${ }^{\circ}$ de núcleos } \\
\hline Ninguno (ref.) & & & & & & & 0,093 & & & 0,039 \\
\hline 1 & & & & & & $1,333(0,910-1,952)$ & 0,140 & 1,582 & $(1,066-2,348)$ & 0,023 \\
\hline 20 más & & & & & & $1,836(1,061-3,177)$ & 0,030 & 1,940 & $(1,098-3,428)$ & 0,023 \\
\hline \multicolumn{11}{|l|}{ Características del entorno } \\
\hline \multicolumn{11}{|l|}{ Clasificación NUTS } \\
\hline Sur y Canarias (ref.) & & & & & & & & & & 0,000 \\
\hline Noroeste & & & & & & & & 1,780 & $(0,960-3,299)$ & 0,067 \\
\hline Noreste & & & & & & & & 0,987 & $(0,493-1,975)$ & 0,971 \\
\hline Comunidad de Madrid & & & & & & & & 2,789 & $(1,523-5,106)$ & 0,001 \\
\hline Centro & & & & & & & & 0,987 & $(0,572-1,704)$ & 0,963 \\
\hline Este & & & & & & & & 2,083 & $(1,284-3,378)$ & 0,003 \\
\hline \multicolumn{11}{|l|}{ Riqueza provincial } \\
\hline Alta (ref.) & & & & & & & & & & 0,098 \\
\hline Media & & & & & & & & 0,627 & $(, 410-, 960)$ & 0,032 \\
\hline Baja & & & & & & & & 0,749 & $(, 473-1,185)$ & 0,217 \\
\hline \multicolumn{11}{|l|}{ Tamaño hábitat } \\
\hline Menos de 5.000 (ref.) & & & & & & & & & & 0,001 \\
\hline Entre 5.000 y 20.000 & & & & & & & & 3,200 & $(1,772-5,779)$ & 0,000 \\
\hline Entre 20.000 y 100.000 & & & & & & & & 2,098 & $(1,75-3,744)$ & 0,012 \\
\hline Más de 100.000 & & & & & & & & 2,054 & $(1,168-3,609)$ & 0,012 \\
\hline \multicolumn{11}{|l|}{ Estado y nivel social del barrio } \\
\hline Zona de clase media-alta (ref.) & & & & & & & & & & 0,000 \\
\hline $\begin{array}{l}\text { Barrio o zona en buenas } \\
\text { condiciones }\end{array}$ & & & & & & & & 2,649 & $(1,351-5,194)$ & 0,005 \\
\hline Barrio o zona deteriorado & & & & & & & & 3,688 & $(1,799-7,563)$ & 0,000 \\
\hline Zona marginal & & & & & & & & 5,600 & $(2,323-13,500)$ & 0,000 \\
\hline $\begin{array}{l}N \text { casos usados por cada } \\
\text { modelo }\end{array}$ & 4.148 & & & 4.112 & & 4.112 & & 4.112 & & \\
\hline$R$ cuadrado de Nagerkelke & 0,019 & & & 0,099 & & 0,102 & & 0,151 & & \\
\hline
\end{tabular}

Fuente: Encuesta Foessa sobre Integración Social y Necesidades Sociales 2013. 
Otra variable que no está incluida en la noción de exclusión pero que guarda con esta una relación muy estrecha es el estado y el nivel social del barrio. Conforme este empeora, la probabilidad de experimentar violencia de género se incrementa, y lo hace de forma muy clara, tanto que, en referencia a las zonas de clase media-alta, en los barrios marginales el riesgo se multiplica por 5,6. Se trata de un resultado especialmente interesante, ya que son muy pocas las investigaciones que se han ocupado de este tema (la gran mayoría de los estudios empíricos existentes contempla únicamente elementos relativos al individuo o al hogar).

El hecho de que el entorno se constituya como un factor de riesgo al margen de la situación del hogar puede remitir a la circunstancia de que, en guetos y zonas marginales, tradicionalmente hayan faltado las herramientas utilizadas para alcanzar una masculinidad exitosa y dominante (cumplir con el rol de proveedor), con lo cual esta habría tendido a redefinirse en términos anómicos (Hampton et al., 2003; Jewkes, 2002). Esto podría, asimismo, haberse acompañado de una progresiva disminución de la reprobación social para con las actitudes violentas de los varones hacia sus parejas y esto, al reducir el miedo a la sanción del entorno, también podría haber contribuido a facilitar el recurso a la violencia (Bandura, 1979).

El segundo modelo de análisis multivariante que se presenta (tabla 6) no incluye la noción de exclusión social globalmente considerada, sino que distingue las diferentes dimensiones que la conforman. Muestra, así, que no todas ellas se asocian con un igual incremento en el riesgo de experimentar violencia de género. Muy al contrario, algunas son muy significativas, otras menos y otras no lo son en absoluto. Más concretamente:

- La dimensión más relevante, con gran distancia con respecto a las demás, es el conflicto social (el riesgo se multiplica aquí por 5,6).

- Otras dimensiones significativas son la exclusión de la educación (el riesgo es un $88 \%$ más elevado) y de la salud (un $57 \%$ más alto).

- La exclusión del empleo, del consumo, de la política, de la vivienda y el aislamiento, por el contrario, no son significativas.

Los resultados obtenidos permiten comprender mejor cómo funciona la relación entre violencia y exclusión. Si antes hemos observado que esta última constituía un factor de riesgo de relevancia bastante reducida, de hecho, ahora comprobamos que eso se debe a que se trata de una noción que reúne en sí situaciones de dificultad muy diferentes, algunas de las cuales constituyen factores de riesgo muy relevantes, mientras que otras no lo son en absoluto. A este respecto, un dato especialmente remarcable es la falta de signifciación de la exclusión del empleo. Este resultado, que se ve refrendado por parte de la literatura (Kiss et al., 2012), sugiere que los elementos de riesgo más significativos no guardan tanta relación con el ámbito económico como con la presencia de conflicto social y, en relación con ello, confirma la oportunidad de haber utilizado, en este análisis, un concepto - como el de exclusión-que, a diferencia de otros comúnmente utilizados, lo contempla. 
Tabla 6. Probabilidad de experimentar violencia de género por parte de la pareja o de la expareja. Modelo que incluye las diferentes dimensiones de la exclusión social. Resumen con las variables significativas

\begin{tabular}{|c|c|c|c|}
\hline & OR & $\mathrm{Cl}$ & $p$ valor \\
\hline \multicolumn{4}{|l|}{ Dimensiones de exclusión } \\
\hline \multicolumn{4}{|l|}{ Dim 4. Exclusión de la educación } \\
\hline \multicolumn{4}{|l|}{ No (ref.) } \\
\hline Sí & 1,876 & $(1,295-2,716)$ & 0,001 \\
\hline \multicolumn{4}{|l|}{ Dim 6. Exclusión de la salud } \\
\hline \multicolumn{4}{|l|}{ No (ref.) } \\
\hline Sí & 1,567 & $(1,183-2,076)$ & 0,002 \\
\hline \multicolumn{4}{|l|}{ Dim 7. Conflicto social } \\
\hline \multicolumn{4}{|l|}{ No (ref.) } \\
\hline Sí & 5,640 & $(3,841-8,282)$ & 0,000 \\
\hline \multicolumn{4}{|l|}{ Características de la mujer } \\
\hline \multicolumn{4}{|l|}{ Discapacidad } \\
\hline \multicolumn{4}{|l|}{ No (ref.) } \\
\hline Sí & 1,849 & $(1,228-2,783)$ & 0,003 \\
\hline \multicolumn{4}{|l|}{ Estado civil } \\
\hline Casada (ref.) & & & 0,000 \\
\hline Pareja de hecho & 1,967 & $(1,313-2,948)$ & 0,001 \\
\hline Separada/divorciada & 5,751 & $(4,184-7,906)$ & 0,000 \\
\hline Viuda & 1,686 & $(0,953-2,982)$ & 0,072 \\
\hline Soltera & 1,549 & $(1,087-2,206)$ & 0,015 \\
\hline \multicolumn{4}{|l|}{ Lugar de origen } \\
\hline \multicolumn{4}{|l|}{ España (ref.) } \\
\hline Otro & 1,506 & $(1,041-2,180)$ & 0,030 \\
\hline \multicolumn{4}{|l|}{ Características del entorno } \\
\hline \multicolumn{4}{|l|}{ Clasificación NUTS } \\
\hline Sur y Canarias (ref.) & & & 0,000 \\
\hline Noroeste & 1,766 & $(0,930-3,355)$ & 0,082 \\
\hline Noreste & 0,933 & $(0,460-1,895)$ & 0,848 \\
\hline Comunidad de Madrid & 2,762 & $(1,486-5,133)$ & 0,001 \\
\hline Centro & 1,130 & $(0,650-1,967)$ & 0,665 \\
\hline Este & 1,989 & $(1,213-3,261)$ & 0,006 \\
\hline \multicolumn{4}{|l|}{ Riqueza provincial } \\
\hline Alta (ref.) & & & 0,092 \\
\hline Media & 0,624 & $(0,401-0,970)$ & 0,036 \\
\hline Baja & 0,685 & $(0,430-1,091)$ & 0,111 \\
\hline \multicolumn{4}{|l|}{ Tamaño hábitat } \\
\hline Menos de 5.000 (ref.) & & & 0,002 \\
\hline Entre 5.000 y 20.000 & 2,954 & $(1,629-5,356)$ & 0,000 \\
\hline Entre 20.000 y 100.000 & 1,873 & $(1,046-3,356)$ & 0,035 \\
\hline Más de 100.000 & 1,765 & $(1,001-3,114)$ & 0,050 \\
\hline \multicolumn{4}{|l|}{ Estado y nivel social del barrio } \\
\hline Zona de clase media-alta (ref.) & & & 0,011 \\
\hline Barrio o zona en buenas condiciones & 2,194 & $(1,121-4,298)$ & 0,022 \\
\hline Barrio o zona deteriorado & 2,860 & $(1,398-5,853)$ & 0,004 \\
\hline Zona marginal & 3,728 & $(1,527-9,097)$ & 0,004 \\
\hline$N$ casos usados por cada modelo & 4.108 & & \\
\hline$R$ cuadrado de Nagerkelke & 0,200 & & \\
\hline
\end{tabular}

Fuente: Encuesta Foessa sobre Integración Social y Necesidades Sociales 2013. 
En lo que respecta a la direccionalidad de la relación encontrada, finalmente, se registran diferencias en función de la dimensión contemplada. En el caso del conflicto social, esta parece claramente ir de la exclusión a la violencia. De los cuatro indicadores contemplados, de hecho, solamente uno (IND 30) identifica situaciones que pueden ser tanto un desencadenante como un resultado de la violencia, mientras que los otros tres hacen referencia a circunstancias que solo pueden haber actuado como desencadenantes. En lo que respecta a la formación, la existencia de una relación (lógica y temporal) de la exclusión a la violencia es aún más evidente. En el caso de la salud, por el contrario, el panorama es diferente, ya que los indicadores correspondientes identifican situaciones que pueden ser tanto anteriores a la violencia como un producto de la misma.

Con el tercer modelo de análisis, que aparece reflejado en la tabla 7 , acercamos ulteriormente la mirada y examinamos los diferentes indicadores a partir de los cuales se construye la variable de exclusión. Observamos así que la probabilidad de experimentar violencia de género se incrementa de forma significativa entre mujeres que viven en hogares en los que:

- Hay personas en instituciones. El riesgo es aquí 13,9 veces más elevado que entre el resto de las mujeres, aunque la escasez de la muestra $(N=15)$ obliga a considerar el dato presentado como únicamente orientativo.

- Las relaciones entre sus miembros son malas. El riesgo es 8,4 veces más elevado que entre el resto de las mujeres, aunque también aquí la muestra es reducida $(N=45)$, lo cual, si bien no pone en duda la significación del dato obtenido, incrementa los márgenes de error.

- Alguien tiene problemas con el alcohol, las drogas o el juego. El riesgo se multiplica por 4,7.

- Se ha pasado hambre. El riesgo se multiplica por 2,4.

- Hay menores de 3 a 15 años sin escolarizar. El riesgo se multiplica por 2,6, aunque la muestra vuelve a ser algo escasa $(N=72)$.

En lo que respecta a la presencia de personas en instituciones (hospitales y pisos psiquiátricos, centros de deshabituación, para menores, para transeúntes, cárceles, etc.), no se encuentran, en la literatura, investigaciones previas que nos ayuden a comprender los resultados obtenidos. Desde aquí, sin embargo, avanzamos la hipótesis de que estos remiten, en primer lugar, al incremento en el riesgo de agresión cuando el varón es drogodependiente o padece alguna enfermedad mental, pero también ponen de relieve la existencia de una asociación entre conflictividad en la esfera pública (manifestada por la presencia de personas en centros penitenciarios y afines) y en la esfera privada. Más concretamente: el hecho de que el varón sea conflictivo y agresivo en la esfera pública, al interseccionarse con un trasfondo estructural de relaciones desiguales de género, puede fácilmente llegar a traducirse en violencia de género en la esfera privada. Finalmente, no podemos olvidar que este indicador identifica situaciones que difícilmente pueden entenderse como el producto de dificulta- 
Tabla 7. Probabilidad de experimentar violencia de género por parte de la pareja o de la expareja. Modelo que incluye los diferentes indicadores de exclusión social. Resumen con las variables significativas

\begin{tabular}{|c|c|c|c|}
\hline & OR & $\mathrm{Cl}$ & $p$ valor \\
\hline \multicolumn{4}{|l|}{ Indicadores de exclusión } \\
\hline \multicolumn{4}{|c|}{ IND 11. Menores de 3 a 15 años no escolarizados } \\
\hline \multicolumn{4}{|l|}{ No (ref.) } \\
\hline Sí & 2,554 & $(1,139-5,728)$ & 0,023 \\
\hline \multicolumn{4}{|l|}{ IND 23. Han pasado hambre } \\
\hline \multicolumn{4}{|l|}{ No (ref.) } \\
\hline Sí & 2,434 & $(1,542-3,840)$ & 0,000 \\
\hline \multicolumn{4}{|l|}{ IND 29. Malas relaciones en el hogar } \\
\hline \multicolumn{4}{|l|}{ No (ref.) } \\
\hline Sí & 8,417 & $(3,232-21,919)$ & 0,000 \\
\hline \multicolumn{4}{|c|}{ IND 30. Problemas con el alcohol, otras drogas o el juego } \\
\hline \multicolumn{4}{|l|}{ No (ref.) } \\
\hline Sí & 4,730 & $(2,840-7,877)$ & 0,000 \\
\hline \multicolumn{4}{|l|}{ IND 35. Personas en instituciones } \\
\hline \multicolumn{4}{|l|}{ No (ref.) } \\
\hline Sí & 13,934 & $(2,983-65,075)$ & 0,001 \\
\hline \multicolumn{4}{|l|}{ Características de la mujer } \\
\hline \multicolumn{4}{|l|}{ Discapacidad } \\
\hline \multicolumn{4}{|l|}{ No (ref.) } \\
\hline Sí & 1,768 & $(1,153-2,711)$ & 0,009 \\
\hline \multicolumn{4}{|l|}{ Estado civil } \\
\hline Casada (ref.) & & & 0,000 \\
\hline Pareja de hecho & 2,353 & $(1,555-3,561)$ & 0,000 \\
\hline Separada/divorciada & 6,361 & $(4,547-8,899)$ & 0,000 \\
\hline Viuda & 1,615 & $(0,898-2,903)$ & 0,110 \\
\hline Soltera & 1,916 & $(1,329-2,762)$ & 0,000 \\
\hline \multicolumn{4}{|l|}{ Lugar de origen } \\
\hline \multicolumn{4}{|l|}{ España (ref.) } \\
\hline Otro & 1,565 & $(1,068-2,294)$ & 0,022 \\
\hline \multicolumn{4}{|l|}{ Nivel educativo } \\
\hline FP grado sup. o universidad (ref.) & & & 0,075 \\
\hline Bachiller o FP grado medio & 1,325 & $(0,880-1,995)$ & 0,177 \\
\hline ESO & 1,485 & $(1,018-2,166)$ & 0,040 \\
\hline Primarios o inferiores & 1,719 & $(1,137-2,598)$ & 0,010 \\
\hline \multicolumn{4}{|l|}{ Características del entorno } \\
\hline \multicolumn{4}{|l|}{ Clasificación NUTS } \\
\hline Sur y Canarias (ref.) & & & 0,000 \\
\hline Noroeste & 1,550 & $(0,789-3,046)$ & 0,203 \\
\hline Noreste & 0,898 & $(0,434-1,858)$ & 0,771 \\
\hline Comunidad de Madrid & 2,767 & $(1,459-5,247)$ & 0,002 \\
\hline Centro & 1,120 & $(0,632-1,985)$ & 0,697 \\
\hline Este & 2,011 & $(1,203-3,360)$ & 0,008 \\
\hline
\end{tabular}


Tabla 7. Probabilidad de experimentar violencia de género por parte de la pareja o de la expareja. Modelo que incluye los diferentes indicadores de exclusión social. Resumen con las variables significativas (continuación)

\begin{tabular}{|c|c|c|c|}
\hline & OR & $\mathrm{Cl}$ & $p$ valor \\
\hline \multicolumn{4}{|l|}{ Características del entorno } \\
\hline \multicolumn{4}{|l|}{ Riqueza provincial } \\
\hline Alta (ref.) & & & 0,044 \\
\hline Media & 0,581 & $(0,373-0,905)$ & 0,016 \\
\hline Baja & 0,646 & $(0,401-1,040)$ & 0,072 \\
\hline \multicolumn{4}{|l|}{ Tamaño hábitat } \\
\hline Menos de 5.000 (ref.) & & & 0,001 \\
\hline Entre 5.000 y 20.000 & 3,078 & $(1,682-5,634)$ & 0,000 \\
\hline Entre 20.000 y 100.000 & 2,012 & $(1,113-3,638)$ & 0,021 \\
\hline Más de 100.000 & 1,772 & $(0,994-3,160)$ & 0,053 \\
\hline \multicolumn{4}{|l|}{ Estado y nivel social del barrio } \\
\hline Zona de clase media-alta (ref.) & & & 0,041 \\
\hline Barrio o zona en buenas condiciones & 2,231 & $(1,113-4,473)$ & 0,024 \\
\hline Barrio o zona deteriorado & 2,747 & $(1,305-5,782)$ & 0,008 \\
\hline Zona marginal & 3,272 & $(1,284-8,337)$ & 0,013 \\
\hline$N$ casos usados por cada modelo & 4.014 & & \\
\hline$R$ cuadrado de Nagerkelke & 0,208 & & \\
\hline
\end{tabular}

Fuente: Encuesta Foessa sobre Integración Social y Necesidades Sociales 2013.

des coyunturales y transitorias, sino que parecen más bien reflejar la presencia de problemáticas mucho más duraderas y enquistadas y que se acompañan, además, de un elevado nivel de estigmatización y marginación social. Este podría ser otro elemento que nos ayude a comprender por qué estas situaciones constituyen un factor de riesgo tan claro.

En lo que se refiere a las malas relaciones en el hogar, los resultados obtenidos confirman los hallazgos de estudios anteriores, que muestran que la presencia de conflictividad en la pareja es un importante factor de riesgo (Aldarondo et al., 2002; Heise, 1998; Jewkes, 2002; Jewkes et al., 2002). En ausencia de encuestas longitudinales, sin embargo, resulta imposible saber si tales mayores niveles de conflictividad preceden a la violencia o la siguen (Jewkes, 2002).

En lo que respecta a los problemas con el alcohol, las drogas y el juego, las características de la fuente no permiten saber si la persona que tiene tales problemas es la mujer o su pareja. Tanto en un caso como en otro, se trata de un hallazgo que corrobora los resultados de estudios anteriores (Barrett et al., 2012; Campbell et al., 2011; Grose y Grabe, 2014; Kiss et al., 2012; Lanier y Maume, 2009; Stöckl et al., 2011).

De modo distinto al supuesto anterior, en lo que se refiere al hecho de haber pasado hambre no se encuentran investigaciones previas. Considerando que se trata de un claro indicador de pobreza y privación, sin embargo, podemos remitirnos a los estudios que vinculan estos dos elementos (Mavrikiou et al., 2014). Observamos así que la privación puede ser tanto un elemento 
detonante — por el estrés y los conflictos que genera (Espinar, 1993; Jewkes, 2002; Walby y Allen, 2004) y por lo que conlleva en términos de masculinidad (Fodor, 2006; Jewkes, 2002; Peralta et al., 2010; Walby y Allen, 2004)_ como una consecuencia de la violencia — por todo lo que esta última implica en términos tanto emocionales como prácticos.

Finalmente, en lo que respecta a la presencia de menores sin escolarizar, tampoco se encuentran referencias empíricas que permitan contrastar los resultados obtenidos ni reflexiones teóricas que consientan comprenderlos. Desde aquí, avanzamos la hipótesis de que, también en este caso, se trata de situaciones que reflejan la existencia de procesos de exclusión claros, intensos y — sobre todo- de largo recorrido y con elevado nivel de enquistamiento, y que este es un primer elemento que nos ayuda a entender los resultados obtenidos. Asimismo, si se considera que un bajo nivel educativo de la mujer también se acompaña de un fuerte incremento en el riesgo de victimización, los datos aquí presentados pueden interpretarse también como una extensión de aquellos.

\subsection{Un análisis diferenciado por tipos de violencia}

Observamos ahora en qué medida la situación de exclusión social incide en el riesgo de experimentar cada tipo de violencia.

Como puede observarse en el gráfico 3, los datos descriptivos indican que la fuerza de la asociación entre violencia y exclusión es máxima en el caso de la violencia física y más reducida en el caso de la violencia sexual y psicológica. Resulta difícil, sin embargo, establecer en cuál de estos dos últimos casos esta es más intensa.

Para obtener una imagen más precisa, se efectúan análisis multivariantes, que permiten aislar el peso del factor exclusión y contemplar —además de la situación social del hogar- también la del entorno, a través de la variable sobre el estado y el nivel social del barrio.

El análisis multivariante, reflejado en la tabla 8, confirma que, en el caso de la violencia física, la asociación con situaciones de dificultad social es máxima: una situación de exclusión moderada, de hecho, se asocia aquí a un aumento del $70 \%$ en la probabilidad de enfrentar violencia; y una de exclusión severa la multiplica por 2,2 (para el conjunto de la violencia de género los incrementos eran del $45 \%$ y del $82 \%$, respectivamente).

La relevancia del factor exclusión se confirma si hacemos referencia a variables de nivel comunitario. Teniendo como referencia a las zonas de clase media-alta, de hecho, en los barrios marginales el riesgo se multiplica por 11,5 (para el conjunto de la violencia de género este se multiplicaba por 5,6).

El menor grado de transversalidad de la violencia física puede guardar relación con dos elementos inextricablemente ligados entre sí. El primero de ellos es el hecho de que, tal y como evidencian los estudios sobre masculinidades (DeKeseredy y Schwartz, 2005), la violencia física es un instrumento que permite a los varones "construir el género", y este instrumento tiende a ser utilizado preferentemente por aquellos que carecen de otros medios que les 
Gráfico 3. Porcentaje de mujeres en situación de integración plena, integración precaria, exclusión moderada y exclusión severa que, en la actualidad, experimentan violencia física, sexual o psicológica por parte de la pareja o de la expareja

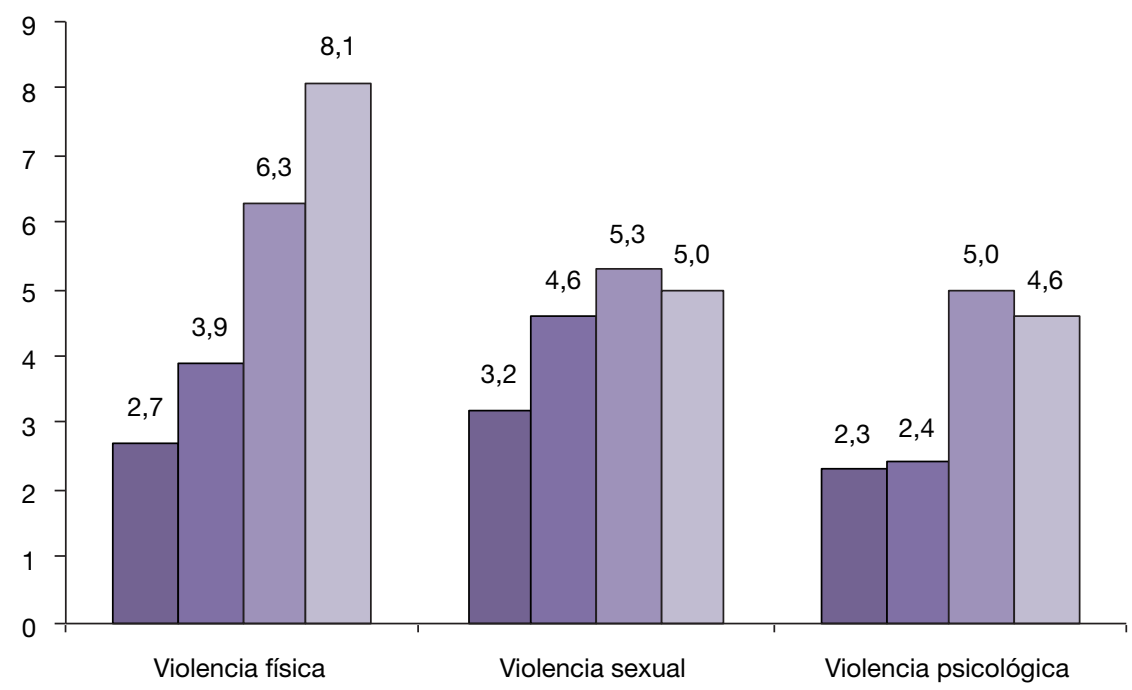

$\square$ Integración plena $\square$ Integración precaria $\square$ Exclusión moderada $\square$ Exclusión severa

Fuente: Encuesta Foessa sobre Integración Social y Necesidades Sociales 2013.

permitan alcanzar una masculinidad dominante y exitosa (una situación especialmente frecuente en situaciones de exclusión social). El segundo elemento es el hecho de que, en situaciones de exclusión, la utilización de la violencia física está más normalizada que en contextos de integración y es, por lo tanto, más fácil que los hombres puedan recurrir a ella. Una última hipótesis que también puede ayudarnos a comprender por qué la asociación entre exclusión y violencia es especialmente elevada en el caso de la violencia física parte de la premisa de que la violencia de género es un proceso gradual y la violencia física el punto de llegada de una escalada en la intensidad del maltrato (Delgado et al., 2007; Fernández, 2004). En este sentido, entonces, el mayor riesgo de violencia física en contextos de exclusión podría simplemente indicar que, allí, la violencia tiende a alcanzar con mayor facilidad unos grados a los que en integración más difícilmente llega.

En el caso de la violencia sexual, también plasmado en la tabla 8, por el contrario, la situación de exclusión social del hogar, globalmente considerada, no es un factor de riesgo significativo (la presencia de una situación de conflicto social, por el contrario, sigue siendo relevante y se asocia a un incremento del $282 \%$ en la probabilidad de experimentar violencia).

El hecho de residir en un barrio marginal, por su parte, se configura aquí como un factor de riesgo muy claro: en referencia a las zonas de clase media- 
Tabla 8. Probabilidad de experimentar violencia física, sexual o psicológica por parte de la pareja o de la expareja. Modelo que incluye la variable de exclusión social

\begin{tabular}{|c|c|c|c|c|c|c|c|c|c|}
\hline & \multicolumn{3}{|c|}{ Física } & \multicolumn{3}{|c|}{ Sexual } & \multicolumn{3}{|c|}{ Psicológica } \\
\hline & $\mathrm{OR}$ & $\mathrm{Cl}$ & $p$ valor & $\mathrm{OR}$ & $\mathrm{Cl}$ & $p$ valor & $\mathrm{OR}$ & $\mathrm{Cl}$ & $p$ valor \\
\hline \multicolumn{10}{|l|}{ Variable de resumen } \\
\hline \multicolumn{10}{|l|}{ Situación de exclusión social } \\
\hline Integración plena (ref.) & & & 0,018 & & & & & & 0,022 \\
\hline Integración precaria & 1,088 & $(0,713-1,659)$ & 0,695 & & & & 0,928 & $(0,581-1,480)$ & 0,753 \\
\hline Exclusión moderada & 1,703 & $(1,025-2,828)$ & 0,040 & & & & 1,912 & $(1,116-3,275)$ & 0,018 \\
\hline Exclusión severa & 2,173 & $(1,195-3,953)$ & 0,011 & & & & 1,630 & $(0,831-3,197)$ & 0,155 \\
\hline \multicolumn{10}{|l|}{ Características de la mujer } \\
\hline \multicolumn{10}{|l|}{ Discapacidad } \\
\hline \multicolumn{10}{|l|}{ No (ref.) } \\
\hline Sí & 1,793 & $(1,058-3,041)$ & 0,030 & 1,727 & $(1,033-2,889)$ & ,037 & & & \\
\hline \multicolumn{10}{|l|}{ Estado civil } \\
\hline Casada (ref.) & & & 0,000 & & & 0,000 & & & 0,000 \\
\hline Pareja de hecho & 1,650 & $(0,926-2,939)$ & 0,089 & 2,090 & $(1,219-3,581)$ & 0,007 & 2,813 & $(1,565-5,053)$ & 0,001 \\
\hline Separada/divorciada & 7,032 & $(4,580-10,798)$ & 0,000 & 6,058 & $(4,028-9,110)$ & 0,000 & 4,993 & $(3,098-8,049)$ & 0,000 \\
\hline Viuda & 2,088 & $(0,954-4,571)$ & 0,065 & 1,825 & $(0,852-3,908)$ & 0,122 & 0,917 & $(0,282-2,979)$ & 0,886 \\
\hline Soltera & 2,226 & $(1,379-3,592)$ & 0,001 & 1,875 & $(1,182-2,974)$ & 0,008 & 1,137 & $(0,615-2,102)$ & 0,682 \\
\hline \multicolumn{10}{|l|}{ Características del entorno } \\
\hline \multicolumn{10}{|l|}{ Clasificación NUTS } \\
\hline Sur y Canarias (ref.) & & & 0,000 & & & & & & 0,000 \\
\hline Noroeste & 3,195 & $(1,102-9,262)$ & 0,032 & & & & 3,095 & $(1,396-6,864)$ & 0,005 \\
\hline Noreste & ,682 & $(0,221-2,105)$ & 0,506 & & & & 17,250 & $(4,332-68,696)$ & 0,000 \\
\hline Comunidad de Madrid & 1,886 & $(0,693-5,135)$ & 0,215 & & & & 24,318 & $(6,572-89,979)$ & 0,000 \\
\hline Centro & 2,559 & $(1,053-6,218)$ & 0,038 & & & & 0,837 & $(0,337-2,081)$ & 0,702 \\
\hline Este & 3,759 & $(1,563-9,042)$ & 0,003 & & & & 2,554 & $(1,327-4,915)$ & 0,005 \\
\hline \multicolumn{10}{|l|}{ Riqueza provincial } \\
\hline Alta (ref.) & & & 0,000 & & & 0,000 & & & 0,000 \\
\hline Media & 0,154 & $(0,083-0,287)$ & 0,000 & 0,208 & $(0,121-0,359)$ & 0,000 & 9,410 & $(2,988-29,635)$ & 0,000 \\
\hline Baja & 0,249 & $(0,128-0,486)$ & 0,000 & 0,150 & $(0,094-0,237)$ & 0,000 & 11,193 & $(3,520-35,588)$ & 0,000 \\
\hline \multicolumn{10}{|l|}{ Tamaño habitat } \\
\hline Menos de 5.000 (ref.) & & & 0,000 & & & 0,000 & & & 0,128 \\
\hline Entre 5.000 y 20.000 & 3,720 & $(1,813-7,633)$ & 0,000 & 3,171 & $(1,574-6,390)$ & 0,001 & 5,519 & $(1,063-28,652)$ & 0,042 \\
\hline Entre 20.000 y 100.000 & 1,989 & $(0,965-4,099)$ & 0,063 & 1,876 & $(0,938-3,751)$ & 0,075 & 6,747 & $(1,352-33,671)$ & 0,020 \\
\hline Más de 100.000 & 1,463 & $(0,730-2,929)$ & 0,283 & 1,313 & $(0,680-2,536)$ & 0,418 & 6,531 & $(1,314-32,455)$ & 0,022 \\
\hline \multicolumn{10}{|l|}{ Estado y nivel social del barrio } \\
\hline Zona de clase media-alta (ref.) & & & 0,003 & & & 0,001 & & & \\
\hline Barrio o zona en buenas condiciones & 3,630 & $(1,267-10,403)$ & 0,016 & 3,418 & $(1,222-9,559)$ & 0,019 & & & \\
\hline Barrio o zona deteriorado & 3,607 & $(1,167-11,145)$ & 0,026 & 4,688 & $(1,601-13,724)$ & 0,005 & & & \\
\hline Zona marginal & 11,468 & $(3,079-42,714)$ & 0,000 & 12,232 & $(3,314-45,150)$ & 0,000 & & & \\
\hline$N$ casos usados por cada modelo & 4.118 & & & 4.101 & & & 4.126 & & \\
\hline$R$ cuadrado de Nagerkelke & 0,211 & & & 0,168 & & & 0,13 & & \\
\hline
\end{tabular}

Fuente: Encuesta Foessa sobre Integración Social y Necesidades Sociales 2013. 
alta, de hecho, allí el riesgo se multiplica por 12,2, un incremento incluso superior al detectado en el caso de la violencia física.

Si se comparan los datos ahora obtenidos con los que hacen referencia a la violencia física, en suma, se deduce que, aquí, la situación del hogar pierde peso a favor de la del entorno. Esto sugiere que el desencadenamiento de violencia sexual no guarda tanta relación con la existencia de dificultades individuales como con la presencia de problemáticas de nivel grupal. Esto, a su vez, permite avanzar la hipótesis de que un incremento en el riesgo de experimentar este tipo de violencia no se deriva tanto de la presencia de elementos estresores como de la modificación (en sentido anómico) de la definición social de masculinidad, un proceso colectivo por definición. Más en detalle, en un contexto, como el de un barrio marginal, en el que los hombres ven mermadas sus posibilidades de encarnar una masculinidad dominante por medios "legítimos», el éxito en el ámbito sexual puede llegar a convertirse en el elemento central de la masculinidad y, en este sentido, la imposición a la pareja de contactos sexuales puede convertirse en una forma de compensación, dirigida a evitar una supuesta emasculación (Peralta et al., 2010).

Finalmente, en el caso de la violencia psicológica, que también aparece reflejado en la tabla 8 , la exclusión social del hogar vuelve a ser un factor de riesgo significativo. Más concretamente, en exclusión moderada la probabilidad de sufrir violencia psicológica se incrementa en un 91\%. En exclusión severa, sin embargo, no se registra un aumento significativo del riesgo. Si se considera que, en el caso de la violencia física, los incrementos eran del $70 \%$ y del $173 \%$, respectivamente, se puede deducir que la exclusión moderada se asocia sobre todo a un aumento en el riesgo de experimentar violencia psicológica y la exclusión severa a un incremento en la posibilidad de experimentar violencia física. Estos datos, en suma, no solamente confirman que la violencia psicológica presenta con situaciones de dificultad social una asociación más débil que otros tipos de violencia, sino que asimismo indican que, cuando esta asociación se da, es sobre todo con las situaciones de dificultad menos intensa.

Asimismo, cabe reseñar que, en este caso y de modo distinto a lo que respecta a la violencia física y sexual, la situación social del barrio no influye de forma significativa en el riesgo de experimentar violencia. Esto confirma que la violencia psicológica presenta una menor asociación con situaciones de dificultad social que otros tipos de violencia.

Esto podría deberse al hecho de que nos enfrentamos, en este caso, a una violencia que es menos evidente que el maltrato físico o sexual, una violencia que puede fácilmente confundirse con comportamientos sexistas (Osborne, 2008) y que es, por ello, mucho más normalizada y, por ende, más transversal que otras formas de maltrato.

\section{Conclusiones}

El estudio aquí efectuado es doblemente novedoso y llena un vacío existente en la literatura específica. Por un lado, de hecho, analiza factores de riesgo de 
violencia de género, pero - de manera distinta a la gran mayoría de estudios de este tipo- lo hace prestando una gran atención a que estos no se confundan con factores causales. En este sentido, subraya que su papel solo puede entenderse en interrelación con el trasfondo estructural de relaciones desiguales de género - este sí causa primaria de la violencia - en el cual se insertan. Por otro, recurre precisamente a la noción de exclusión social, no a los factores de riesgo habitualmente utilizados en la literatura especializada. Esta noción, además, se mide con gran riqueza y precisión y con atención al factor ecológico, a través de las variables sobre estado y nivel social del barrio. Esto último resulta especialmente relevante, ya que la producción científica que se engloba bajo el planteamiento ecológico se ha concretado sobre todo en estudios de carácter teórico, mientras que apenas se ha traducido en investigaciones de carácter empírico como esta (De Alencar-Rodrígues y Cantera, 2012).

Esta investigación, además de novedosa, es fuertemente necesaria. El hecho de que la violencia sea un fenómeno estructural y transversal (que, como tal, puede afectar a mujeres pertenecientes a todo el espectro social), en efecto, no puede llevarnos a ignorar el mayor riesgo de victimización al que se enfrentan las mujeres en situación de exclusión. Esto implica que el hecho de que, desde posturas feministas, se siga rechazando cualquier explicación más allá de la que remite a la dominación masculina — aunque puede ser hasta útil para la causa de todas aquellas mujeres que no acumulan más opresiones que la de género (es el caso de mujeres blancas, autóctonas, de clase media-alta, con elevado nivel formativo, etc.) - es claramente contraproducente para aquellas que ocupan posiciones más precarizadas, y esto no es algo que, desde posiciones progresistas, se pueda defender.

Hemos demostrado que, en situaciones de exclusión, el riesgo de experimentar violencia de género aumenta. Al descomponer los procesos de exclusión en las dimensiones que los conforman, sin embargo, hemos descubierto asimismo que este riesgo se incrementa sobre todo en presencia de procesos anómicos, relacionados con la conflictividad a nivel tanto social como de hogar (cuando el riesgo llega a multiplicarse por 5,6), mientras que otras dimensiones —entre ellas una situación de exclusión económica — no parecen influir. Este resultado pone de manifiesto la importancia y la oportunidad de haber analizado aquí la relación entre violencia y exclusión, en lugar de hacerlo entre violencia y pobreza (como es más habitual).

Tales hallazgos nos llevan a recomendar la introducción, en las encuestas que se ocupan de violencia de género, de preguntas que permitan acercarse a la dimensión social. En este sentido, la incorporación de una pregunta sobre ingresos en la Macroencuesta de Violencia contra la Mujer 2015 debe interpretarse positivamente, pero es claramente insuficiente, sobre todo si se considera que los elementos de riesgo más significativos no guardan relación tanto con el ámbito económico como con la presencia de conflicto social.

A todo esto se añade que no es solamente la situación de dificultad social del hogar lo que se asocia con una mayor probabilidad de sufrir violencia de género, sino también (y sobre todo) la de la zona de residencia (en referencia 
a las zonas de clase media-alta, en los barrios marginales el riesgo se multiplica por 5,6), un resultado que confirma la importancia de considerar elementos de nivel comunitario (algo que, en la actualidad, apenas se hace).

Hemos demostrado que, en contextos de exclusión, el riesgo de victimización se incrementa. En ausencia de encuestas longitudinales, sin embargo, es imposible determinar con exactitud en qué medida tal situación de exclusión es preexistente a la violencia o un producto de la misma (aunque la mayoría de los indicadores contemplados sugieren un recorrido de la exclusión a la violencia). Es esta una limitación común a la práctica totalidad de los estudios sobre factores de riesgo y que solo podrá resolverse con la introducción de encuestas tipo panel (Barrett et al., 2012; Campbell et al., 2011; Friedemann-Sánchez y Lovatón, 2012; Stöckl et al., 2011).

Una vez examinada la violencia de género en su conjunto, hemos asimismo diferenciado por tipo de violencia, un análisis que realiza únicamente una pequeña minoría de los estudios sobre factores de riesgo consultados (Friedemann-Sánchez y Lovatón, 2012; Grose y Grabe, 2014; Khalifeh, et al. 2013; Nagassar et al., 2010; Ruiz-Pérez et al., 2006). De esta manera, hemos podido concluir que si, en lo que atañe a la violencia física y sexual, la situación de desventaja comparativa de las mujeres excluidas aparece con total claridad, en lo que respecta a la violencia psicológica esta es mucho más reducida. Se trata de unos resultados muy relevantes, que ponen de relieve que, aunque hallarse en una situación de dificultad social es un factor de riesgo para determinados tipos de maltrato, no lo es para todos, sino que existen también tipologías de violencia que son auténticamente transversales y tienden a afectar por igual a mujeres pertenecientes a todo el espectro social.

En relación con esto, cabe asimismo resaltar que, aunque la probabilidad de experimentar violencia de género es mucho mayor en el espacio de exclusión, 7 de cada 10 víctimas son mujeres en situación de integración social (plena o precaria). Nuevamente, esto nos recuerda que nos enfrentamos a un fenómeno que tiene carácter estructural y cuyos orígenes han de ser buscados en el nivel de la estructura social patriarcal en la que nos movemos y que, como tal, puede afectar a mujeres de toda clase y circunstancia social (aunque no a todas en la misma medida).

\section{Referencias bibliográficas}

AA. VV. (2014). VII Informe sobre exclusión y desarrollo social en España. Madrid. Foessa.

AldARONDO, E. y CASTRO-FERnÁNDEZ, M. (2011). «Risk and protective factors for domestic violence perpetration». En: WHITE J.W.; Koss, M.P. y KAZDIN, A.E. (eds.). Violence against women and children: Mapping the terrain. Vol. 1. Washington: American Psychological Association. $<$ https://doi.org/10.1037/12307-010>

AldARONDO, E.; KAUfMAN, G. y JASINSKI, L. (2002). «A Risk Marker Analysis of Wife Assault in Latino Families». Violence Against Women, 8 (4), 429-454. <https://doi.org/10.1177/107780120200800403> 
Alencar-Rodrígues, R. de y Cantera, L. (2012). «Violencia de género en la pareja: Una revisión teórica». Psico $\Psi, 43$ (1), 116-126.

AlmeidA, R. y LOCKARD, J. (2005). «The cultural context model». En: SOKOLOFF, N.J. y PrATt, C. (eds.). Domestic violence at the margins: Readings in race, class, gender, and culture. Piscataway, Nueva Jersey: Rutgers University Press.

AtKinson, M. P.; Greenstein, T. N. y LANG, M. M. (2005). «For women, breadwinning can be dangerous: Gendered resource theory and wife abuse». Journal of Marriage and the Family, 67 (5), 1137-1148. <https://doi.org/10.1111/j.1741-3737.2005.00206.x>

BANDURA, A. (1979). «The social learning perspective: Mechanisms of aggression». En: Toch, H. (ed.). Psychology of crime and criminal justice. Nueva York: Holt, Reinehart \& Winston.

Barrett, B.J.; Habibov, N. y Chernyak, E. (2012). «Factors Affecting Prevalence and Extent of Intimate Partner Violence in Ukraine: Evidence From a Nationally Representative Survey». Violence Against Women, 18 (10), 1147-1176. <https://doi.org/10.1177/1077801212464387>

Basile, K.C.; Hall, J.E. y Walters, M.L. (2013). «Expanding Resource Theory and Feminist-Informed Theory to Explain Intimate Partner Violence Perpetration by Court-Ordered Men». Violence Against Women, 19 (7), 848-880. <https://doi.org/10.1177/1077801213497105>

BERKOWITZ, L. (1989). «Frustration-aggression hypothesis: Examination and reformulation». Psychological Bulletin, 106, 59-73. <https://doi.org/10.1037/0033-2909.106.1.59>

Bograd, M. (2005). «Stregthening Domestic Violence Theories: Intersections of Race, Class, Sexual Orientation, and Gender». En: SoKoloff, N.J. (ed.). Domestic Violence at the Margins: Reedings on Race, Class, Gender, and Culture. Nueva Jersey: Rutgers University Press.

BOON, B. y FARnsworTh, J. (2011). «Social Exclusion and Poverty: Translating Social Capital into Accessible Resources». Social Policy and Administration, 45 (5), 507-524. <https://doi.org/10.1111/j.1467-9515.2011.00792.x>

Bourdieu, P. (1980). El sentido práctico. Madrid: Taurus, 1991.

- (1998). La dominación masculina. Barcelona: Editorial Anagrama, 2000.

BRUGUÉ, Q.; GOMÁ, R. y SubirATs, J. (2002). «De la pobreza a la exclusión social: Nuevos retos para las políticas públicas». Revista Internacional de Sociología, 33, 337-345.

Cabrera, P.J. (2004). «Pobreza y exclusión desde la perspectiva de género». En: GARCía-Mina Freire, A. y Carrasco Galán, M.J. (eds.). Género y desigualdad: La feminización de la pobreza. Madrid: Publicaciones de la Universidad Pontificia Comillas.

CALLÁ, R.M. (2008). «Gestione violenta dei conflitti nella coppia: Risultati di un'integrazione teórica». En: CORRADI, C. (ed.). I modelli sociali della violenza contro le donne. Milán: Franco Angeli.

Campbell, J.C.; Alhusen, J.; Draughon, J.; Kub, J. y Walton-Moss, B. (2011). «Vulnerability and protective factors for intimate partner violence». En: WHITE, J.W.; Koss, M.P. y KazDin, A.E. (eds.). Violence against women and children: Mapping the terrain. Vol. 1. Washington: American Psychological Association. <https://doi.org/10.1037/12307-011>

CASTEL, R. (1995). Las metamorfosis de la cuestión social: Una crónica del salariado. Buenos Aires: Paidós, 1997. 
CHAN, K.L. (2014). «Assessing the Risk of Intimate Partner Violence in the Chinese Population: The Chinese Risk Assessment Tool for Perpetrator (CRAT-P)». Violence Against Women, 20 (5), 500-516. $<$ https://doi.org/10.1177/1077801214535107>

Choo, H.Y. y Ferree, M.M. (2010). «Practicing Intersectionality in Sociological Research: A Critical Analysis of Inclusions, Interactions, and Institutions in the Study of Inequalities». Sociological Theory, 28 (2), 129-149. <https://doi.org/10.1111/j.1467-9558.2010.01370.x>

Chung, G.H.; TUCKer, M.B. y TAKEUCHI, D. (2008). «Wives’ relative income production and household male dominance: Examining violence among Asian American enduring couples». Family Relations, 57 (2), 227-238. <https://doi.org/10.1111/j.1741-3729.2008.00496.x>

Coker, A.L.; Williams, C.M.; Follingstad, D.R. y Jordan, C.E. (2011). «Psychological, reproductive and maternal health, behavioral, and economic impact of intimate partner violence». En: WHITE, J.W.; Koss, M.P. y KAZDIN, A.E. (eds.). Violence against women and children: Mapping the terrain. Vol. 1. Washington: American Psychological Association. <https://doi.org/10.1037/12307-012>

Collins, P.H. (1990-2000). Black Feminist Thought: Knowledge, Consciousness, and the Politics of Empowerment. Boston: Unwin Hyman.

CORRADI, C. (2008). «Introduzione: I modelli sociali della violenza contro le donne». En: CORRADI. C. (ed.). I modelli sociali della violenza contro le donne. Milán: Franco Angeli.

Cunradi, C.B.; Ames, G.M. y Duke, M. (2011). "The relationship of alcohol problems to risk for unidirectional and bidirectional intimate partner violence among a sample of blue-collar couples». Violence and Victims, 26 (2), 147-158. $<$ https://doi.org/10.1891/0886-6708.26.2.147>

Cunradi, C.B.; AMES, G.M. y MoOre, R.S. (2008). «Prevalence and Correlates of Intimate Partner Violence Among a Sample of Construction Industry Workers». Journal of Family Violence, 23, 101-112.

<https://doi.org/10.1007/s10896-007-9131-x>

Cunradi, C.B.; Caetano, R.; Clark, C. y Schafer, J. (2000). «Neighborhood Poverty as a Predictor of Intimate Partner Violence Among, White, Black and Hispanic Couples in the Unites States: A Multilevel Analysis». Annals of Epidemiology, 10 (5), 297-308. <https://doi.org/10.1016/S1047-2797(00)00052-1>

Damant, D.; Lapierre, S.; Kouraga, A.; Fortin, A.; Hamelin-Brabant, L.; Lavergne, C. y Lessard, G. (2008). «Taking Child Abuse and Mothering Into Account». Journal of Women and Social Work, 23 (2). $<$ https://doi.org/10.1177/0886109908314321>

Daoud, N.; Smylie, J.; Urquia, M.; Allan B. y O'Campo, P. (2013). "The Contribution of Socio-economic Position to the Excesses of Violence and Intimate Partner Violence Among Aboriginal Versus Non-Aboriginal Women in Canada». Revue Canadienne de Santé Publique, 104 (4), 278-283. <https://doi.org/10.17269/cjph.104.3724>

DeKeseredy, W.S. y SCHWARTZ, M.D. (2005). «Masculinities and Interpersonal Violence». En: Kimmel, M.S.; Hearn, J. y Connell, R.W. (eds.). Handbook of Studies on Men and Masculinities. California: Sage Publications. <https://doi.org/10.4135/9781452233833.n20> 
Delgado Álvarez, C.; Iraegui Torralba, A.; Marquina Torres, L.; Martín Tabernero, M. F.; Palacios Vicario, B.; Plaza Sánchez, J. F. y Sánchez GÓmEZ, M.C. (2007). «Patrones de masculinidad y feminidad asociados al ciclo de la violencia de género». Revista De Investigación Educativa, RIE, 25 (1), 187-218.

Dollard, J.; Doob, L.W.; Miller, N.E.; Mowrer; O.H. y SEArs, R.R. (1939). Frustration and aggression. New Haven (CT): Yale University Press, 1967. <https://doi.org/10.1037/10022-000>

Elley, S. (2011). «Young Women, Class and Gendered Heterosexuality: The Implication of Educational Aspirations and Social Networks for Sex Education Messages». Sociology, 45 (3), 413-429. <https://doi.org/10.1177/0038038511399621>

EsPinar RuIZ, E. (1993). Violencia de género y procesos de empobrecimiento: Estudio de la violencia contra las mujeres por parte de su pareja o ex pareja sentimental. Alicante: Universidad de Alicante. Tesis doctoral.

FALCI, C. (2011). «Self-esteem and mastery trajectory in high school by social class and gender». Social Sciences Research, 40 (2), 586-601. <https://doi.org/10.1016/j.ssresearch.2010.12.013>

FERnÁNDEZ Villanueva, C. (2004). «Violencia contra las mujeres: Una visión estructural». Psychosocial Intervention, 13 (2), 155-164.

Ferrer Pérez, V.A.; Bosch Fiol, E. y Riera Madurell, M.T. (2006). «Las dificultades en la cuantificación de la violencia contra las mujeres en la pareja: Análisis psicosocial». Psychosocial Intervention, 15 (2), 181-202. <https://doi.org/10.4321/S1132-05592006000200005>

Fodor, E. (2006). «A Different Type of Gender Gap: How Women and Men Experience Poverty». East European Politics and Societies, 20 (1), 14-39. $<$ https://doi.org/10.1177/0888325405284248>

Fox, G.L.; Benson, M.; DeMARIs, A.A. y VANWYK, J. (2002). «Economic distress and intimate violence: Testing family stress and resources theories». Journal of Marriage o The Family, 64, 793-807. <https://doi.org/10.1111/j.1741-3737.2002.00793.x>

Franklin, C.A. y MenaKer, T.A. (2014). «Feminism, Status Inconsistency, and Women's Intimate Partner Victimization in Heterosexual Relationships». Violence Against Women, 20 (7), 825-845. <https://doi.org/10.1177/1077801214543385>

Friedemann-SÁnchez, G. y Lovatón, R. (2012). «Intimate Partner Violence in Colombia: Who Is at Risk?». Social Forces, 91 (2) 663-688. $<$ https://doi.org/10.1093/sf/sos131>

Galtung, J. (1990). Violencia cultural. Guernika-Lumo: Gernika Gogoratuz, 2003.

GANDARIAS GOIKOETXEA, I. (2016). En marcha hasta que todas seamos libres: Encuentros, tensiones y retos para la construcción de articulaciones entre organizaciones de mujeres migradas y feministas en Euskal Herria. Barcelona: Universitat Autònoma de Barcelona. Tesis doctoral.

García Selgas, Fernando J. y Casado Aparicio, E. (2010). Violencia en la pareja: Género y vínculo. Madrid: Talasa.

GonzÁlez, R. y Santana, J.D. (2001). Violencia en parejas jóvenes: Análisis y prevención. Madrid: Pirámide.

Gonzalez-Guarda, R.M.; Vermeesch, A.L.; Florom-Smith, A.L.; Mccabe, B.E. y Peragallo, N.P. (2013). «Birthplace, Culture, Self-Esteem, and Intimate Part- 
ner Violence Among Community-Dwelling Hispanic Women». Violence Against Women, 19 (1), 6-23. $<$ https://doi.org/10.1177/1077801212475336>

Grose, R.G. y Grabe, S. (2014). «The Explanatory Role of Relationship Power and Control in Domestic Violence Against Women in Nicaragua: A Feminist Psychology Analysis». Violence Against Women, 20 (8), 972-993. $<$ https://doi.org/10.1177/1077801214546231>

HambY, S.L. (2005). «The Importance of Community in a Feminist Analysis of Domestic Violence among Native Americans». En: SoKOLOFF, N.J. y DUPONT, I. (eds.). Domestic Violence at the Margins: Reedings on Race, Class, Gender, and Culture. Nueva Jersey: Rutgers University Press.

Hampton, R.; Oliver, W. y Magarian, L. (2003). "Domestic Violence in the African American Community». Violence Against Women, 9 (5), 533-577. $<$ https://doi.org/10.1177/1077801202250450>

HeISE, L.L. (1998). «Violence Against Women: An Integrated, Ecological Framework». Violence Against Women, 4, 262-290. <https://doi.org/10.1177/1077801298004003002>

Hochschild, A. (1991). The Second Shift. Nueva York: Penguin.

- (2009). La mercantilización de la vida intima: Apuntes de la casa y el trabajo. Argentina: Rústica.

IZQUIERDO BENITO, M.J. (2007). «Estructura y acción en la violencia de género». En: MOLAS FONT, M.D. (ed.). Violencia deliberada: Las raices de la violencia patriarcal. Barcelona: Icaria.

JeWKes, R. (2002). «Intimate partner violence: Causes and prevention». The Lancet, 359, 1423-1429. <https://doi.org/10.1016/S0140-6736(02)08357-5>

JeWKes, R.; LEVIN, J. y PENN-KeKANA, L. (2002). «Risk factors for domestic violence: Findings from a South African cross-sectional survey». Social Science and Medicine, 55 (9), 1603-1617. <https://doi.org/10.1016/S0277-9536(01)00294-5>

JosEPHSON, J. (2005). «The Intersectionality of Domestic Violence and Welfare in the Lives of Poor Women». En: SoKoloff, N.J. y Dupont, I. (eds.). Domestic Violence at the Margins: Reedings on Race, Class, Gender, and Culture. Nueva Jersey: Rutgers University Press.

KhalifeH, H.; Hargreaves, J.; Howard, L.M. y Birdthistle, I. (2013). «Intimate Partner Violence and Socioeconomic Deprivation in England: Findings from a National Cross-Sectional Survey». American Journal of Public Health, 103 (3), 462-472. <https://doi.org/10.2105/AJPH.2012.300723>

Kiss, L.; Schraiber, L.B.; Heise, L.; Zimmerman, C.; Gouveia, N. y Watts, C. (2012). «Gender-based violence and socioeconomic inequalities: Does living in a more deprived neighborhoods increase women's risk of intimate partner violence?». Social Science and Medicine, 74 (8), 1172-1179. <https://doi.org/10.1016/j.socscimed.2011.11.033>

Kwan, P. (1997). "Intersections of Race, Ethnicity, Class, Gender \& Sexual Orientation: Jeffrey Dahmer and the Cosynthesis of Categories». Hastings Law Journal, 48, 1257-1293.

LAGARDE Y DE lOS Ríos, M. (2008). Amor y sexualidad, una mirada feminista. Curso de verano. Universidad Menéndez Pelayo. 
Lanier, C. y Maume, M. (2009). «Intimate Partner Violence and Social Isolation Acros the Rural/Urban Divide». Violence Against Women, 15 (11), 1311-1330. <https://doi.org/10.1177/1077801209346711>

LAPARRA, M. (2010). «El impacto de la crisis en la cohesión social o el surf de los hogares españoles en el modelo de integración de la sociedad líquida». Documentación Social, 168, 97-130.

Laparra, M.; Obradors, A.; Pérez, B.; Pérez Yruela, M.; Renes, V.; Sarasa, S.; SubiRATS. J. y TRUjILlo, M. (2007). «Una propuesta de consenso sobre el concepto de exclusión: Implicaciones metodológicas». Revista Española del Tercer Sector, 5, 15-57.

LAPARRA, M.; Zugasti, N. y GARCÍA LAUTRE, I. (2014). «Reflexiones metodológicas en el análisis de la exclusión social». VII Informe sobre exclusión y desarrollo social en España. Madrid: Foessa. Documento de trabajo.

LAWSON, J. (2012). «Sociological Theories of Intimate Partner Violence». Journal of Human Behavior in the Social Environment, 22 (5), 572-590. <https://doi.org/10.1080/10911359.2011.598748>

Mavrikiou, P.M.; Apostolidou, M. y Parlalis, S.K. (2014). «Risk factors for the prevalence of domestic violence against women in Cyprus». The Social Science Journal, 51 (2), 295-301. <https://doi.org/10.1016/j.soscij.2014.02.002>

Merton, R.K. (1968). Teoría y estructura sociales. México: Fondo de Cultura Económica, 1980.

Messerschmidt, J.W. (1993). Masculinities and Crime: Critique and Reconceptualization of Theory. Maryland: Rowman \& Littlefield.

Montañés, P. y Moyano, M. (2006) «Violencia de género sobre inmigrantes en España: Un análisis psicosocial». Pensamiento Psicológico, 2 (6), 21-32.

Nagassar, R.P.; Rawlins, J.M.; SAMPSOn, N.; Zackerali, J.; ChanKadyal, K.; Ramasir, C. y BoOdram, R. (2010). «The Prevalence of Domestic Violence within Different Socio-economic Classes in Central Trinidad». West Indian Medical Journal, 59 (1), 20-25.

NiXON, J. y HuMPHREYS, C. (2010). «Marshalling the Evidence: Using Interseccionality in the Domestic Violence Frame». Social Politics, 17 (2), 137-158. $<$ https://doi.org/10.1093/sp/jxq003>

Osborne Verdugo, R. (2008). «De la "violencia” (de género) a las "cifras de la violencia”: Una cuestión política». Empiria: Revista De Metodología De Ciencias Sociales, $15,99-124$.

<https://doi.org/10.5944/empiria.15.2008.1201>

OxOBY, R. (2009). "Understanding social inclusion, social cohesion, and social capital». International Journal of Social Economics, 36 (12), 1133-1152. $<$ https://doi.org/10.1108/03068290910996963>

Panchanadeswaran, S.; Ting, L.; Burke, J.G.; O'Campo, P.; Mcdonnel, K. y Gielen, A.C. (2010). «Profiling Abusive Men Based on Women's Self-Reports: Finding From a Sample of Urban Low-Income Minority Women». Violence Against Women, 16 (3), 313-327. $<$ https://doi.org/10.1177/1077801209360854>

Peralta, R.L.; TutTle, L.A. y Steele, J. (2010). «At the Intersection of Interpersonal Violence, Masculinity, and Alcohol Use: The Experiences of Heterosexual Male Perpetrators of Intimate Partner Violence». Violence Against Women, 16 (4), 387-409. <https://doi.org/10.1177/1077801210363539> 
PIRANI, E. (2011). «Evaluating contemporary social exclusion in Europe: A hierarchical latent class approach». Quality \& Quantity: International Journal of Methodology, 47 (2), 923-941. <https://doi.org/10.1007/s11135-011-9574-2>

PRINS, B. (2006). «Narrative Accounts of Origins: A Blind Spot in the Intersectional Approach?». European Journal of Women's Studies, 13 (3), 277-290. <https://doi.org/10.1177/1350506806065757>_.

Raj, A. y Silverman, J. (2002). "Violence Against Immigrant Women». Violence Against Women, 8 (3), 367-398. <https://doi.org/10.1177/10778010222183107>

RamireZ, I.L. (2007). "The Relationship of Acculturation and Social Integration to Assaults on Intimate Partners Among Mexican American and Non Mexican White Students». Journal of Family Violence, 22, 533-542. <https://doi.org/10.1007/s10896-007-9102-2>

RENNER, L.M. y WHITNEY, S.D. (2012). «Risk factors for unidirectional and bidirectional intimate partner violence among young adults». Child Abuse and Neglect, 36 (1), 40-52. <https://doi.org/10.1016/j.chiabu.2011.07.007>

Richie, B. (2000). «A Black feminist reflection on the antiviolence movement». Signs, 25, $1133-1137$. <https://doi.org/10.1086/495533>

Riger, S. y StaggS, S.L. (2004). "Welfare Reform, Domestic Violence and Employment». Violence Against Women, 10 (9), 961-990. <https://doi.org/10.1177/1077801204267464>

RodrígueZ-Borrego, M.-A.; VAquero-Abellán, M. y Bertagnolli da Rosa, L. (2012). «Estudio transversal sobre factores de riesgo de sufrir violencia por compañero íntimo en la mujer enfermera». Revista Latino-Americana de Enfermagem, 20 (1), 1-8.

Ruiz-Pérez, I.; Plazaola-Castaño, J.; Álvarez-Kindelán, M.; Palomo-Pinto, M.; Arnalte-Barrera, M.; Bonet-Pla, Á.; Santiago-Hernando, M.L. de; Herranz-Torrubiano, A.; Garralón-Ruiz, L.M. y Gender Violence Study Group (2006). «Sociodemographic Associations of Physical, Emotional, and Sexual Intimate Partner Violence in Spanish Women». Annals of Epidemiology, $6(5), 357-363$.

<https://doi.org/10.1016/j.annepidem.2005.03.007>

Santo, J.B.; Bukowski, W.M.; Stella-Lopez, L.; Carmago, G.; Mayman, S.B. y ADAMS, R.E. (2013). «Factors Underlying Contextual Variations in the Structure of the Self: Differences related to SES, Gender, Culture, and "Majority/Nonmajority" Status During Early Adolescence». Journal of Research on Adolescence, 23 (1), 69-80. <https://doi.org/10.1111/j.1532-7795.2012.00793.x>

SMITH, M.D. (1990). "Patriarchal Idelology and Wife Beating: A Test of the Feminist Hypothesis». Violence and Victims, 5 (4), 257-273.

SMiTH, D.L. (2008). «Disability, Gender and Intimate Partner Violence: Relationships from the Behavioural Risk Factor Surveillance System». Sexuality and Disability, 26 (1), 15-28. <https://doi.org/10.1007/s11195-007-9064-6>

SMith, C.A.; Ireland, T.O.; PARK A.; ElWyn, L. y Thornberry, T.P. (2011). «Intergenerational Continuities and Discontinuities in Intimate Partner Violence: A 
Two-Generational Prospective Study». Journal of Interpersonal Violence, 26 (18), 3720-3752. $<$ https://doi.org/10.1177/0886260511403751>

SoKoloff, N.J. (2004). «Domestic Violence at the Crossroads: Violence Against Poor Women and Women of Color». Women's Study Quarterly, 32 (3/4), 139-147.

SoKoloff, N.J. y DupOnT, I. (2005). «Domestic Violence at the Intersections of Race, Class and Gender». Violence Against Women, 11 (1), 38-64. <https://doi.org/10.1177/1077801204271476>

Stith, S.M.; Smith, D.B.; PenN, C.E.; Ward, D.B. y TriTt, D. (2004). «Intimate partner physical abuse perpetration and victimization risk factors: A meta-analytic review». Aggression and Violent Behavior, 10 (1), 65-98. <https://doi.org/10.1016/j.avb.2003.09.001>

STÖCKL, H.; HeISE, L. y WATTS, C. (2011). «Factors associated with violence by a current partner in a nationally representative sample of German women». Sociology of Health and Illness, 33 (5), 694-709. <https://doi.org/10.1111/j.1467-9566.2011.01319.x>

STRIER, R.; SigAD, L.; EIsIKOVITZ, Z. y BuChBINDER, E. (2014). «Masculinity, Poverty and Work: The Multiple Constructions of Work among Working Poor Men». Journal of Social Policy, 43 (2), 331-349. <https://doi.org/10.1017/S0047279413000949>

Subirats, J. (dir.) (2004). Pobreza y exclusión social. Un análisis de la realidad española y europea. Barcelona: Fundación "la Caixa".

- (2005). Análisis de los factores de exclusión social. Bilbao: Fundación BBVA.

TAUCHEN, H. y WiTTE, A.D. (2001). «The dynamics of domestic violence». Effect of Institutions on Economic Behavior, 85, 414-418.

Uthman, O.A.; MORADI, T. y LaWOKO, S. (2009). «The independent contribution of individual-, neighbourhood-, and country-level socioeconomic position on attitudes towards intimate partner violence against women in sub-Saharan Africa: A multilevel model of direct and moderating effects». Social Science \& Medicine, 68 (10), 1801-1809. $<$ https://doi.org/10.1016/j.socscimed.2009.02.045>

Van Bergen, A.; Hoff, S.; Van Ameijden, E. y Van Hemer, A. (2014). «Measuring Social Exclusion in Routine Public Health Surveys: Construction of a Multidimensional Instrument». Plos One, 9 (5), 1-11. <https://doi.org/10.1371/journal.pone.0098680>

VAN WIJK, N.Ph.L. y De Bruijn, J.G.M. (2012). «Risk Factors for Domestic Violence in Curacao». Journal of Interpersonal Violence, 27 (15), 3032-3053. <https://doi.org/10.1177/0886260512441074>

Veselska, Z.; Madarasova Geckova, A.; Gajdosova, B.; Orosova, O.; Van Dijk, J.P. y ReijneVElD, S.A. (2010). "Socio-economic differences in self-esteem of adolescents influenced by personality, mental health and social support». European Journal of Public Health, 20 (6), 647-652. <https://doi.org/10.1093/eurpub/ckp210>

VillaViCENCiO, P. (1993). «Mujeres maltratadas: Conceptualización y evaluación». Clinica y Salud, 4 (3), 215-228.

Vives-Cases, C.; Álvarez-Dardeta, C.; Gil-González, D.; TorrubianoDOMíNGUEZ, J.; RohlFs, I. y EsCRIBÀ-AGÜIR, V. (2009). «Perfil sociodemográfico de las mujeres afectadas por violencia del compañero íntimo en España». Gaceta Sanitaria, 23 (5), 410-414. <https://doi.org/10.1016/j.gaceta.2009.02.007> 
Vives-Cases, C.; Gil-GonzÁlez, D.; Ruiz-Pérez, I.; EscribÀ-AgÜIr, V.; PlazaOla-Castaño, J.; Montero-Piñar, M.I. y Torrubiano-Domínguez, J. (2010). «Identifying sociodemographic differences in Intimate Partner Violence among immigrant and native women in Spain: A cross-sectional study». Preventive Medicine, 51 (1), 85-87.

$<$ https://doi.org/10.1016/j.ypmed.2010.03.017>

Walby, S. y Allen, J. (2004). Domestic violence, sexual assault and stalking: Findings from the British Crime Survey. Londres: Home Office Research, development and Statistics Directorate.

WARnER, L.R. y SHIELDS, S.A. (2013). «The Intersections of Sexuality, Gender and Race: Identity Research at the Crossroads». Sex Roles, 68 (11/12), 803-810. <https://doi.org/10.1007/s11199-013-0281-4>

YICK, A. (2001). «Feminist Theory and Status Inconsistency Theory». Violence Against Women, 7 (5), 545-562. <https://doi.org/10.1177/10778010122182596>

Zhang, L. y POSTIGLIONE, G.A. (2001). «Thinking Styles, self-esteem, and socio-economic status». Personality and Individual Differences, 31 (8), 1333-1346. <https://doi.org/10.1016/S0191-8869(00)00227-0> 
